Three-dimensional distortions of the tokamak plasma boundary: boundary displacements in the presence of resonant magnetic perturbations

This content has been downloaded from IOPscience. Please scroll down to see the full text.

2014 Nucl. Fusion 54083006

(http://iopscience.iop.org/0029-5515/54/8/083006)

View the table of contents for this issue, or go to the journal homepage for more

Download details:

IP Address: 128.178.125.173

This content was downloaded on 20/08/2014 at 09:35

Please note that terms and conditions apply. 


\title{
Three-dimensional distortions of the tokamak plasma boundary: boundary displacements in the presence of resonant magnetic perturbations
}

\author{
I.T. Chapman ${ }^{1}$, M. Becoulet ${ }^{2}$, T. Bird ${ }^{3}$, J. Canik ${ }^{4}$, M. Cianciosa ${ }^{5}$, W.A. Cooper ${ }^{6}$, T. Evans ${ }^{7}$, \\ N. Ferraro ${ }^{7}$, C. Fuchs ${ }^{8}$, M. Gryaznevich ${ }^{1}$, Y. Gribov ${ }^{9}$, C. Ham ${ }^{1}$, J. Hanson ${ }^{5}$, G. Huijsmans ${ }^{9}$, \\ A. Kirk ${ }^{1}$, S. Lazerson ${ }^{10}$, Y. Liang ${ }^{11}$, I. Lupelli ${ }^{1}$, R.A. Moyer ${ }^{12}$, C. Nührenberg ${ }^{3}$, F. Orain ${ }^{2}$, D. Orlov ${ }^{12}$, \\ W. Suttrop ${ }^{8}$, D. Yadykin ${ }^{13}$, the ASDEX Upgrade, DIII-D, MAST and NSTX Teams and EFDA-JET \\ Contributors $^{\mathrm{a}}$
}

${ }^{1}$ EURATOM/CCFE Fusion Association, Culham Science Centre, Abingdon, Oxon, OX14 3DB, UK

${ }^{2}$ CEA, IRFM, F-13108 Saint-Paul-lez-Durance, France

${ }^{3}$ Max Planck Institute for Plasma Physics, EURATOM Association, Wendelsteinstr. 1, 17491 Greifswald, Germany

${ }^{4}$ Oak Ridge National Laboratory, Oak Ridge, TN 37831-8071, USA

${ }^{5}$ Physics Department, Auburn University, Auburn, AL 36849, USA

${ }^{6}$ CRPP, Association EURATOM/Confédération Suisse, EPFL, 1015 Lausanne, Switzerland

${ }^{7}$ General Atomics, PO Box 85608, San Diego, CA 92186, USA

${ }^{8}$ Max-Planck-Institut fur Plasmaphysik, EURATOM-Association D-85748 Garching, Germany

${ }^{9}$ ITER Organization, Route de Vinon sur Verdon, 13115 St Paul Lez Durance, France

${ }^{10}$ PPPL, Princeton University, PO Box 451, Princeton, NJ 08543, USA

${ }^{11}$ Forschungszentrum Jülich GmbH, IEF-4, 52425 Jülich, Germany

${ }^{12}$ University of California, San Diego, La Jolla, CA 92093-0417, USA

${ }^{13}$ EURATOM/VR Association, Chalmers University, Gothenburg, Sweden

E-mail: ian.chapman@ccfe.ac.uk

Received 13 November 2013, revised 23 December 2013

Accepted for publication 31 January 2014

Published 21 May 2014

\begin{abstract}
The three-dimensional plasma boundary displacements induced by applied non-axisymmetric magnetic perturbations have been measured in ASDEX Upgrade, DIII-D, JET, MAST and NSTX. The displacements arising from applied resonant magnetic perturbations (RMPs) are measured up to $\pm 5 \%$ of the minor radius in present-day machines. Good agreement can be found between different experimental measurements and a range of models - be it vacuum field line tracing, ideal three-dimensional MHD equilibrium modelling, or nonlinear plasma amplification. The agreement of the various experimental measurements with the different predictions from these models is presented, and the regions of applicability of each discussed. The measured displacement of the outboard boundary from various machines is found to correlate approximately linearly with the applied resonant field predicted by vacuum modelling (though it should be emphasized that one should not infer that vacuum modelling accurately predicts the displacement inside the plasma). The RMP-induced displacements foreseen in ITER are expected to lie within the range of those predicted by the different models, meaning less than $\pm 1.75 \%( \pm 3.5 \mathrm{~cm})$ of the minor radius in the $\mathrm{H}$-mode baseline and less than $\pm 2.5 \%( \pm 5 \mathrm{~cm})$ in a $9 \mathrm{MA}$ plasma. Whilst a displacement of $7 \mathrm{~cm}$ peak-to-peak in the baseline scenario is marginally acceptable from both a plasma control and heat loading perspective, it is important that ITER adopts a plasma control system which can account for a three-dimensional boundary corrugation to avoid an $n=0$ correction which would otherwise locally exacerbate the displacement caused by the applied fields.
\end{abstract}

Keywords: boundary displacement, resonant magnetic perturbation, non-axisymmetry

(Some figures may appear in colour only in the online journal)

\footnotetext{
a See the appendix of Romanelli et al 2012 Proc. 24th Int. Conf. on Fusion Energy 2012 (San Diego, CA, 2012) (Vienna: IAEA) www-pub.iaea.org/iaeameetings/41985/24th-Fusion-Energy-Conference.
} 


\section{Introduction and background}

When tokamak plasmas operate in a high-confinement regime, the plasma edge is usually susceptible to quasi-periodic instabilities called edge localized modes (ELMs) [1]. These ELMs are understood to be a manifestation of so-called peeling-ballooning instabilities driven by strong pressure gradients and localized current density at the edge of the plasma [2,3]. ELMs can eject potentially damaging levels of energy and particles from the confined plasma, hence affecting the lifetime of plasma facing components [4]. In order to ensure a an appropriate lifetime of plasma facing components in ITER a robust ELM control scheme is required which either suppresses the ELMs completely or, at least, reduces the heat flux per ELM which is incident on the divertor plates [5]. One such control scheme is the application of resonant magnetic perturbations (RMPs), which perturb the magnetic field in the edge transport barrier, or pedestal, region. RMPs have been applied to completely suppress ELMs in DIII-D [6,7] and KSTAR [8]—in ASDEX Upgrade [9, 10], DIII-D [12], MAST [12-14] and JET $[15,16]$. Although control of ELMs by applying RMPs clearly involves imposing a non-axisymmetric perturbation to the magnetic field, the plasma is still often treated two dimensionally in equilibrium reconstruction and stability analyses [7, 17-21].

However, previous results [22-29] have shown that the application of RMPs can cause significant non-axisymmetric distortions to the plasma boundary. Such perturbations to the boundary could lead to unacceptable heat loads on the plasma facing components in ITER. For $15 \mathrm{MA} Q=10$ operation reducing the separatrix distance from the reference position ( $15 \mathrm{~cm}$ from the first wall) to less than $6 \mathrm{~cm}$ leads to the power flux on the ITER Beryllium panels to exceed their design limit of $4.7 \mathrm{MWm}^{-2}$. These power fluxes have been modelled taking into account parallel transport of the energy in the edge plasma during the stationary phases and the ELMs (using the model of [31]) as well as the 3D structure of the wall and the filamentary structure of the ELMs [32]. Control of the plasma position is required to maintain stationary ICRH coupling; the accuracy of which depends on the steepness of the ITER edge density profiles in the far scrape-off layer. For steep far SOL density profiles the maximum ICRH coupled power decreases/increases at constant applied voltage to the antenna by a factor of 2 when the plasma position is moved away/closer to the antenna by $4 \mathrm{~cm}$. Therefore, for these density profiles, stationary ICRH coupling requires that the separatrix position is kept within a range of at most $\pm 2 \mathrm{~cm}$ [33]. Consequently it is important that we can predict the likely distortions in ITER and prepare methods for the avoidance or control of such boundary displacements.

Past experiments to measure the plasma perturbation when non-axisymmetric perturbation fields are applied have been performed on DIII-D [22, 28-30], ASDEX Upgrade [23-25], MAST [26, 27] and JET [26, 34]. In most cases, applied fields from either ex- or in-vessel correction coils had a demonstrable and significant effect on the location of the plasma boundary, deforming the separatrix by a few percent of the minor radius. In a similar vein, experiments in JET and JT-60U with varying toroidal field ripple were shown to affect the plasma pedestal and edge behaviour $[35,36]$.
Measurements of the displacements caused by applying non-axisymmetric magnetic perturbations in ASDEX Upgrade, DIII-D, JET, MAST and NSTX are presented in section 2. In section 3, these measurements are compared to numerical modelling, either treating the field perturbation using just the vacuum field approximation, including an ideal plasma response, or a resistive plasma response. Finally, predictions for the displacements expected in ITER due to externally applied RMPs are made in section 4, before the implications are discussed in section 5.

\section{Measurements of displacements due to RMPs in present machines}

The effect of applying perturbations with different toroidal mode number has been investigated in MAST double null diverted (DND) plasmas optimized for diagnostic coverage. The primary diagnostics used to measure the radial position of the edge of the plasma are: the linear $D_{\alpha}$ camera, the phantom colour camera, the charge exchange recombination spectroscopy diagnostic, the RGB camera, a charge-coupled device (CCD) camera, the reflectometer and the Thomson scattering diagnostic, all of which measure the boundary in different toroidal positions. Since there are twelve lower invessel coils in MAST, the phase of the $n=3$ applied field can easily be changed in $30^{\circ}$ quanta. In order to maximize the measurable perturbation of the edge of the plasma, two phases of an $n=3 \mathrm{RMP}$ were applied with $60^{\circ}$ between them. This has the added benefit that the position controller does not significantly correct for the distortion due to the applied RMPs as it is constrained by measurements of the plasma position in a sector experiencing a null in displacement for both phases of applied field. The radial position of the plasma boundary for six different high resolution diagnostics when the two phases of RMPs are applied (compared to a reference plasma in the absence of non-axisymmetric applied fields) is shown in figure 1 [27]. The expected toroidal phase dependence (with arbitrary amplitude) is added to guide the eye. It is clear that not only is there a different dependence of the edge corrugation in the two phases of the applied field, the toroidal variation follows the expected $n=3$ periodicity symptomatic of the RMP applied. Furthermore, the amplitude of the displacement is found to be in good agreement on a number of different diagnostics and is approximately $\pm 1.5 \mathrm{~cm}$, which represents more than $\pm 2.5 \%$ of the minor radius [27].

A similar dependence is found when two phases of an $n=4$ RMP field are applied, with figure 2 showing a clear $n=4$ toroidal periodicity, again with an amplitude of toroidal corrugation of approximately $\pm 1.5 \mathrm{~cm}$. In figure 1 the position of the outboard midplane when RMPs are not applied is found to be in good agreement adding credence to the toroidal corrugation measured with RMPs.

Measurement of edge displacements have also been made in JET when $n=2$ RMPs are applied from the set of ex-vessel error field correction coils (EFCCs) [34]. The direct measurements of the plasma boundary displacement are obtained using high resolution Thomson scattering (HRTS) diagnostic by following the time evolution of the edge density profile. The procedure to determine the pedestal position takes into consideration the HRTS instrument function as described 


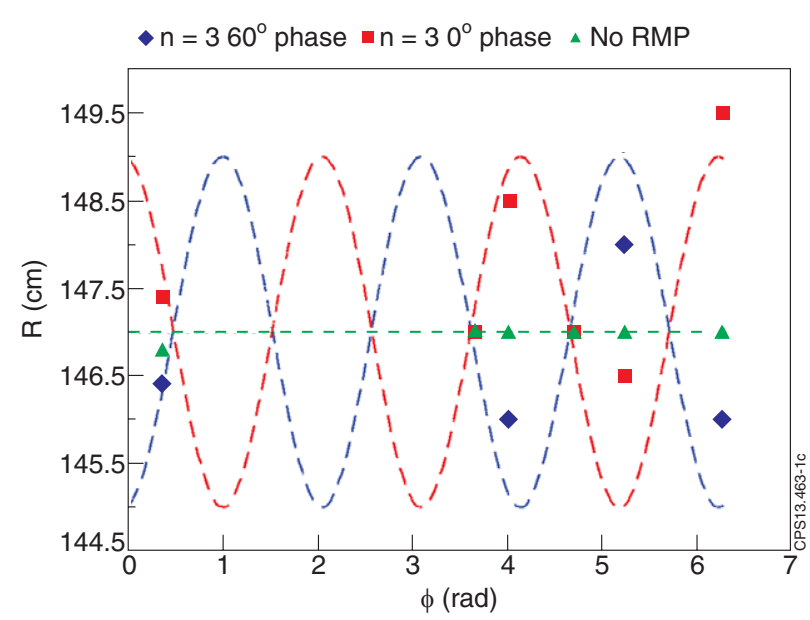

Figure 1. MAST. The midplane boundary position as a function of toroidal angle as measured by six different diagnostics with sub-cm radial resolution in MAST plasmas with two phases of an applied $n=3$ RMP applied. The dashed line is added with $n=3$ periodicity and arbitrary amplitude to guide the eye.

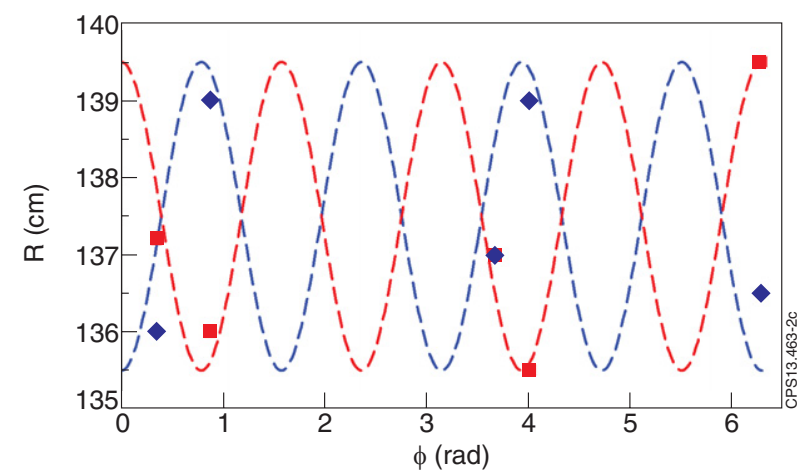

Figure 2. MAST. The midplane boundary position as a function of toroidal angle as measured by six different diagnostics with sub-cm radial resolution in MAST plasmas with two phases of an applied $n=4$ RMP applied. The dashed line is added with $n=4$ periodicity and arbitrary amplitude to guide the eye.
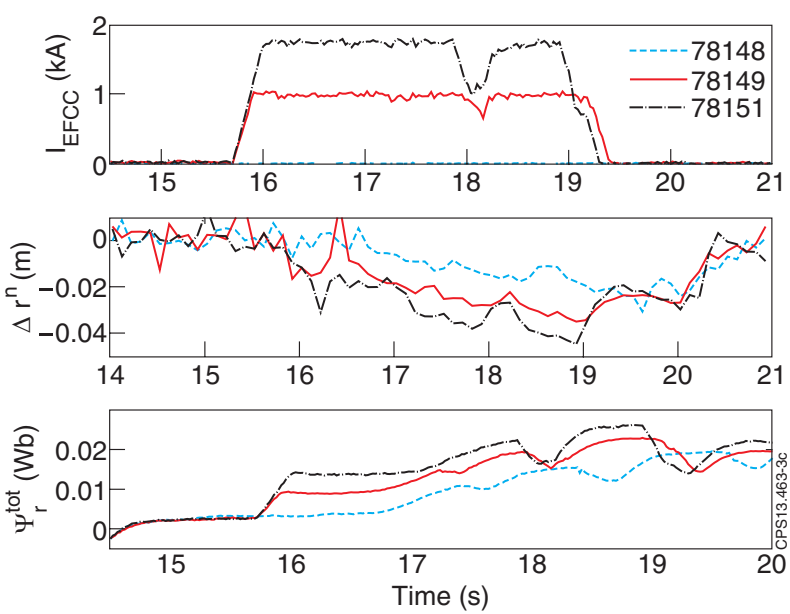

Figure 3. JET. The RMP-induced displacement measured by the Thomson scattering diagnostic as a function of time (centre) compared to the current in the ex-vessel coils in JET (top) and change in the magnetic flux (bottom).

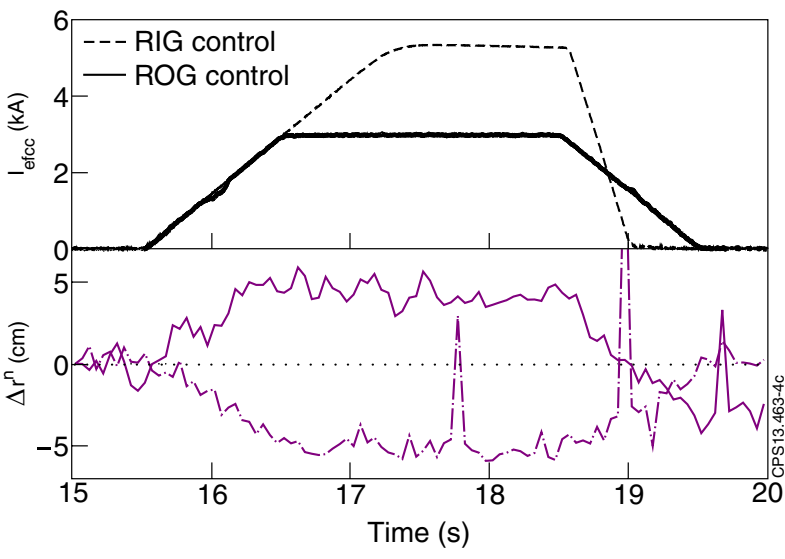

Figure 4. JET. The RMP-induced displacement measured by the Thomson scattering diagnostic as a function of time compared to the current in the ex-vessel coils in JET. The plasma position control is either done on the gap between the plasma boundary and the inner wall (dashed) or the outer wall (solid).

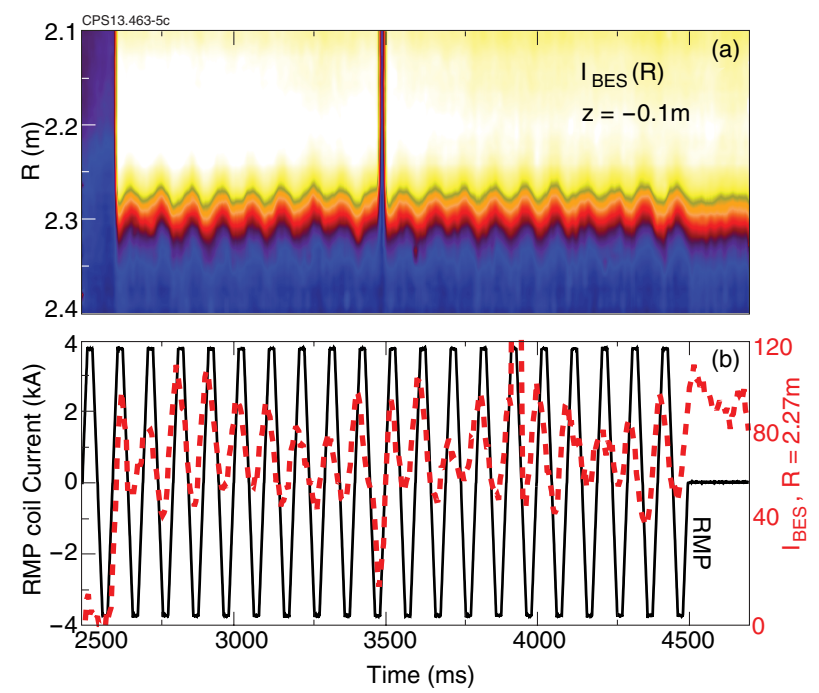

Figure 5. DIII-D. (a) Colour contours of the beam emission versus major radius $R$ at an elevation of $z=0.1 \mathrm{~m}$ on the outer midplane of the DIII-D tokamak during the RMP pulse. The rotating $n=2$ field starts at $2250 \mathrm{~ms}$ (before the start of the plot), showing a coherent oscillation in phase with the rotating RMP. (b) Evolution of the rotating $n=2$ RMP coil current in the upper $150^{\circ}$ segment (thin black line), and the measured beam emission intensity (thick red dashed line) at $R=2.27 \mathrm{~m}$ and $z=0.1 \mathrm{~m}$. The beam emission in the edge is modulated by the rotating $n=2$ RMP. Reproduced with permission from Moyer R.A. et al 2012 Nucl. Fusion 52123019. Copyright 2012 IAEA Vienna.

in [37]. The time evolution of the EFCC current, plasma boundary displacement measured by the HRTS (scaled to have zero displacement before application of the EFCC produced magnetic field) and the radial magnetic flux measured by the coincident flux loop for the shots with different values of the EFCC current are shown in figure 3. The effect of the field produced by EFCC coils on the plasma boundary is clearly seen in the kinetic measurements. Note a rapid transient drop in the EFCC current occurred at $t=18 \mathrm{~s}$ and this reduction in the applied field is manifest as a reduction in the edge distortion, illustrating the dependence of the edge displacement on the applied field strength. 


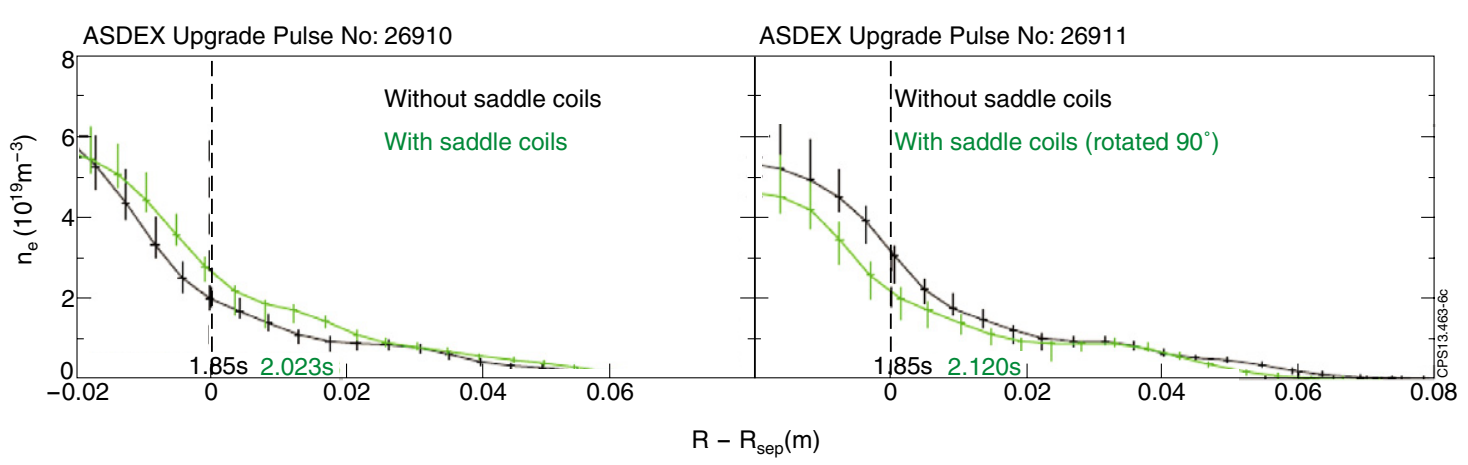

Figure 6. ASDEX Upgrade density profiles from the lithium beam as a function of the distance to the unperturbed separatrix at the height of the beam at time points between ELMs shortly before (black) and after (green) switching on the saddle coils. The two pictures show shots with the magnetic perturbation rotated by $90^{\circ}$.

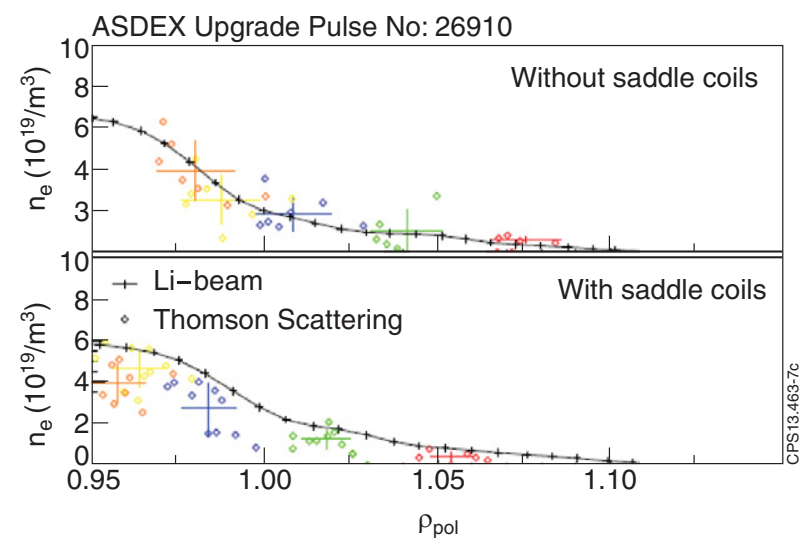

Figure 7. ASDEX Upgrade mapping of the density measurements from Li-beam (black) and Thomson scattering (coloured, each colour denotes one channel) on the normalized poloidal flux radius. Thomson scattering measurements are averaged over $100 \mathrm{~ms}$, using only time points between ELMs.

The response of the plasma controller, whilst not studied in detail, is striking. Figure 4 shows the measured displacement in JET when the plasma position feedback uses either the gap between the last closed flux surface and the magnetic loops on the centre column, or the outer gap between the plasma and the low-field side flux loops. It is evident that the plasma control system induces a shift in opposite directions depending upon the position feedback scheme employed. The difference between these two schemes is up to $10 \mathrm{~cm}$. This exemplifies the acute need to account for non-axisymmetric displacements in the plasma position control feedback system.

The response of the plasma boundary and separatrix to a $10 \mathrm{~Hz} n=2$ rotating RMP has been studied on DIIID using active imaging of the Doppler-shifted Balmer $D_{\alpha}$ emission from high energy injected neutrals (beam emission spectroscopy, BES) [29]. The measurements were obtained in ELMing H-mode discharges with an ITER-similar shape and pedestal collisionality, $v_{, \mathrm{e}} \sim 0.3$, similar to pedestal conditions predicted for ITER. The internal RMP coils generated an $n=2$ even parity RMP rotating counter to the plasma current in the toroidal direction at $10 \mathrm{~Hz}$. The temporal evolution of the measured beam emission at an elevation of $Z=0.1 \mathrm{~m}$ is plotted in figure 5 as a function of major radius $R$. A coherent oscillation is clearly seen in the radius of the steep gradient

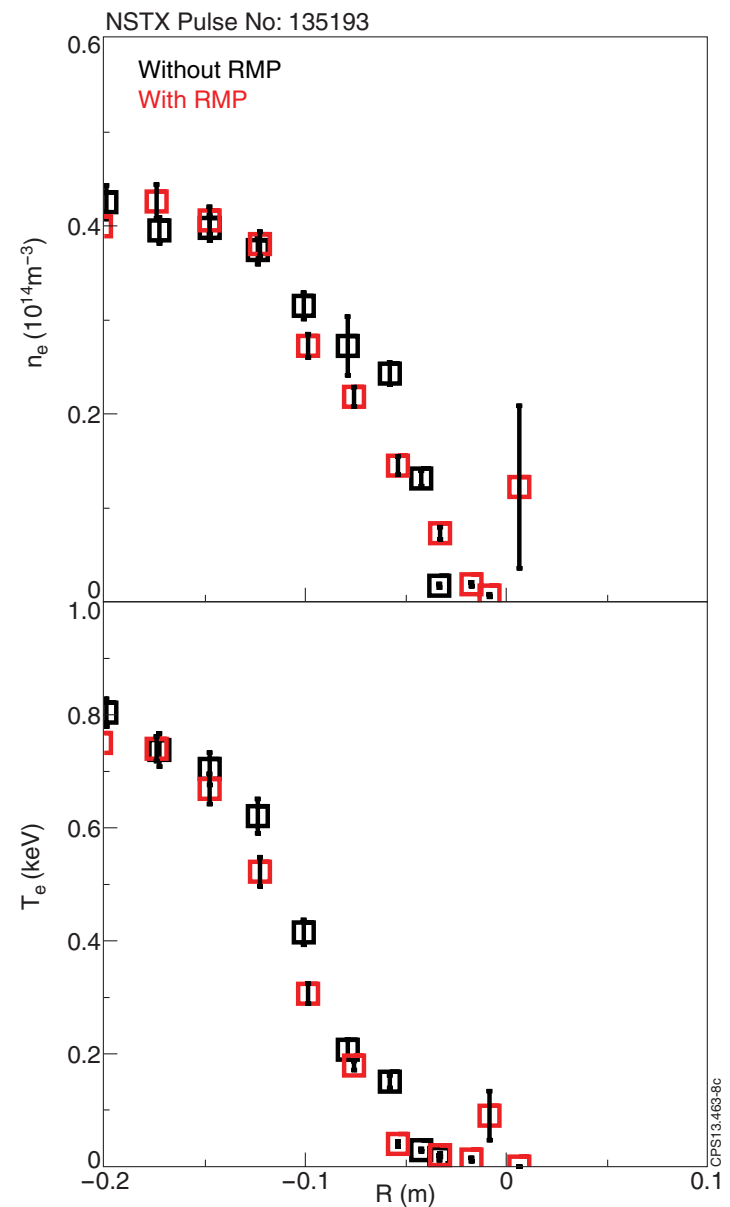

Figure 8. NSTX. Thomson scattering measurements of the electron density and temperature profiles in NSTX with and without an $n=3$ applied RMP, showing negligible displacement caused by the RMP.

region of the profile near $R=2.30 \mathrm{~m}$. In figure 5 , the variation in the beam emission at $(R, Z)=(2.27,0.1)$ is plotted versus time alongwith the RMP coil current. The beam emission intensity (thick, dashed red line) oscillates coherently and is phase locked with the rotating $n=2$ RMP (thin solid black line), with an amplitude of $\pm 1 \mathrm{~cm}$, equivalent to $\pm 1.6 \%$ of the minor radius [29].

The variation in the electron density profile at an elevation of $Z=0.05 \mathrm{~m}$ on the LFS midplane has also been measured by 
profile reflectometry in the same DIII-D discharge [29]. There is a clear coherent oscillation in the major radius $R$ of the steep edge gradient region of the profile as the $n=2$ perturbation is rotated toroidally past the diagnostic which is quantitatively similar to the displacement measured in the beam emission intensity profile. This displacement in the major radius of the steep edge gradient region is on the order of $\pm 1 \mathrm{~cm}$, or $\pm 1.7 \%$ [29] of the minor radius.

ASDEX Upgrade have also made measurements of the edge displacements when RMPs are applied [23-25]. A series of identical discharges with $1 \mathrm{MA}$ plasma current, a toroidal magnetic field of $-2.4 \mathrm{~T}$, fractional Greenwald density of $n_{\mathrm{e}} / n_{\mathrm{GW}} \sim 0.64,9.7 \mathrm{MW}$ additional heating and $1 \mathrm{kA}$ in-vessel coil current has been performed, where only the configuration of the in-vessel coils has been varied: 2 discharges with mode number $n=2$, odd up/down-parity (i.e. opposite polarity of upper and lower coils at the same toroidal position), with zero and $90^{\circ}$ toroidal orientation of the magnetic perturbations, and one discharge with $n=2$, even parity. The density profile measured by the lithium beam diagnostic just before and after the saddle coils have been switched on is shown in figure 6 . For an otherwise identical discharge where the configuration of the saddle coils has been rotated by $90^{\circ}$ the shift of the perturbed separatrix and correspondingly the shift in the density profile goes in the opposite direction.

Figure 7 shows edge density profiles from the Li-beam and Thomson scattering diagnostics mapped onto the poloidal flux radius $\rho_{\text {pol }}$. Whereas the mapping is good without RMPs, the profiles from the two diagnostics seem to be shifted apart when the coils are switched on. This is mostly due to the fact that the toroidal positions of the two diagnostics differ by $137^{\circ}$ and see a different shift of the perturbed separatrix, in this case outward for the Li-beam and inward for the Thomson scattering diagnostic. The displacement between these two sectors, which should see the maximum displacement for an $n=2$ field, is only $\pm 3 \mathrm{~mm}$, which is equivalent to $0.6 \%$ of the minor radius [25], significantly less than in DIII-D, JET and MAST.

A similarly negligible displacement is observed in NSTX when an $n=3$ RMP is applied from the ex-vessel coils [40], as seen in figure 8 . Here, the electron density and temperature profiles are shown (averaged for many time slices both with and without RMPs applied) as measured by the Thomson scattering diagnostic. The displacement is well within the error bars of the diagnostic, so the RMPs have no discernible effect on the boundary.

Overall, there is a wide range of displacements observed in different machines: NSTX and ASDEX Upgrade observe sub-cm displacements for all configurations of applied fields. In contrast, MAST, DIII-D and JET can measure significant displacements, up to $\pm 3.5 \%$ of the minor radius when the field is resonant. Furthermore, the amplitude, and even the direction of the boundary displacement is sensitively dependent upon the interaction of the plasma response to the applied nonaxisymmetric field and the radial position feedback control system.

\subsection{Multi-machine database}

In order to compare the measurements of non-axisymmetric displacements made in different machines, a multi-machine

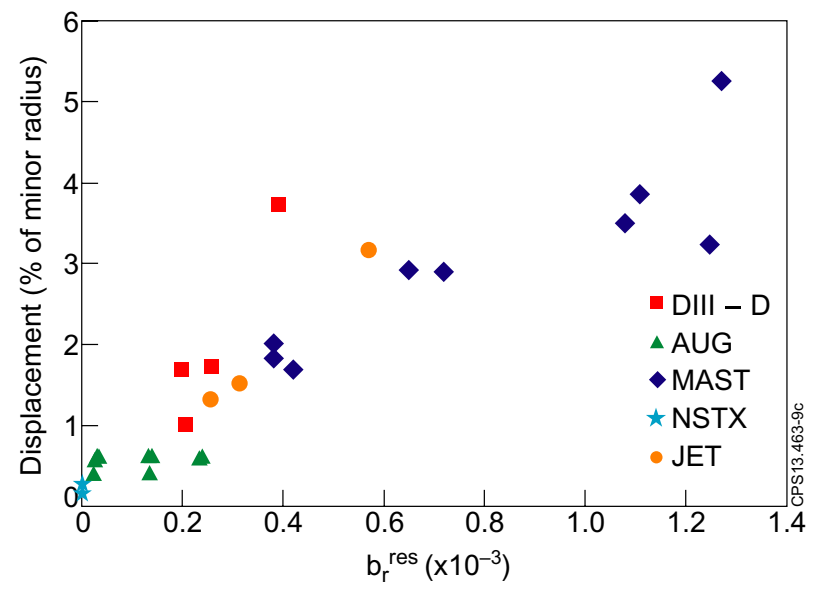

Figure 9. The RMP-induced displacement as a fraction of the minor radius as a function of the resonant applied field at the last rational surface as defined in equation (1) for a multi-machine database covering a wide range of $q_{95}$, RMP toroidal mode number and spectrum, and $\beta_{\mathrm{N}}$.

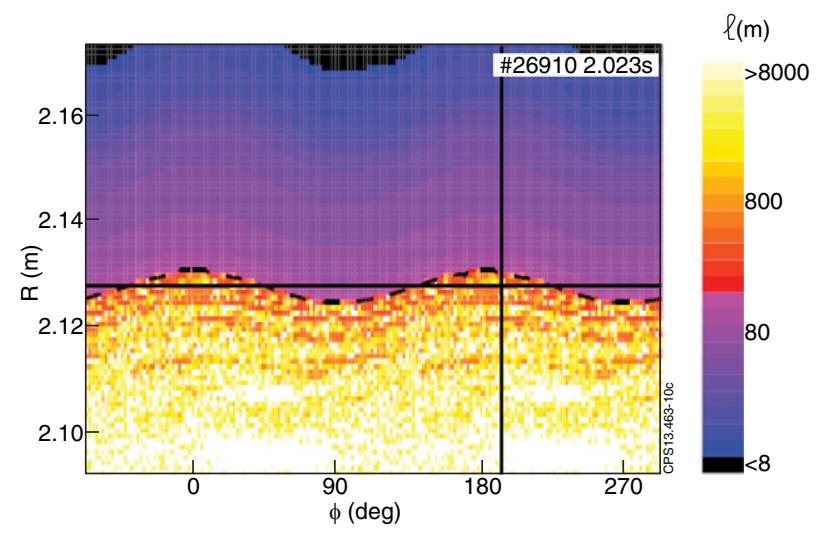

Figure 10. ASDEX Upgrade. The plasma boundary predicted by a field line tracing calculation as a function of toroidal angle for ASDEX Upgrade shot 26910 with an $n=2$ RMP applied, showing the perturbed boundary compared to the position of the separatrix before RMPs are applied (solid black line). Connection length to the low field side target for field lines starting at a horizontal plane at $z=0.326 \mathrm{~m}$ (height of the lithium beam) around the torus. Marked are the position of the lithium beam at $193^{\circ}$ and the unperturbed separatrix at $R=2.125 \mathrm{~m}$. The dotted line marks the position of the 'perturbed separatrix'.

database has been established, incorporating data from 30 discharges from 5 different machines. The database contains information on $I_{\mathrm{p}}, B_{\mathrm{t}}, R_{0}, a, q_{95}, n_{\mathrm{e}, \mathrm{ped}} / n_{\mathrm{GW}}, \beta_{\mathrm{N}}, b_{\mathrm{res}}^{\mathrm{r}}$ and the measured displacement. There is a very weak correlation between the displacement and all other parameters except for the resonant applied field. For this study, a universal definition of the resonant field is used. Here, $b^{1}$ represents the normalized component of the perturbed field perpendicular to equilibrium flux surfaces and is given by $b^{1}=\left(B \cdot \nabla \sqrt{\psi_{\text {pol }}}\right) /(B \cdot \nabla \phi)$ where $B$ is the total field vector and $\phi$ is the toroidal angle [38]. The effective radial resonant field component of the applied perturbation normalized to the toroidal field $\left(b_{\text {res }}^{\mathrm{r}}\right)$ is the amplitude of the resonant Fourier component in the spectrum of $b^{1}$ divided by the average value of $\nabla \sqrt{\psi_{\mathrm{pol}}}$ on the magnetic 

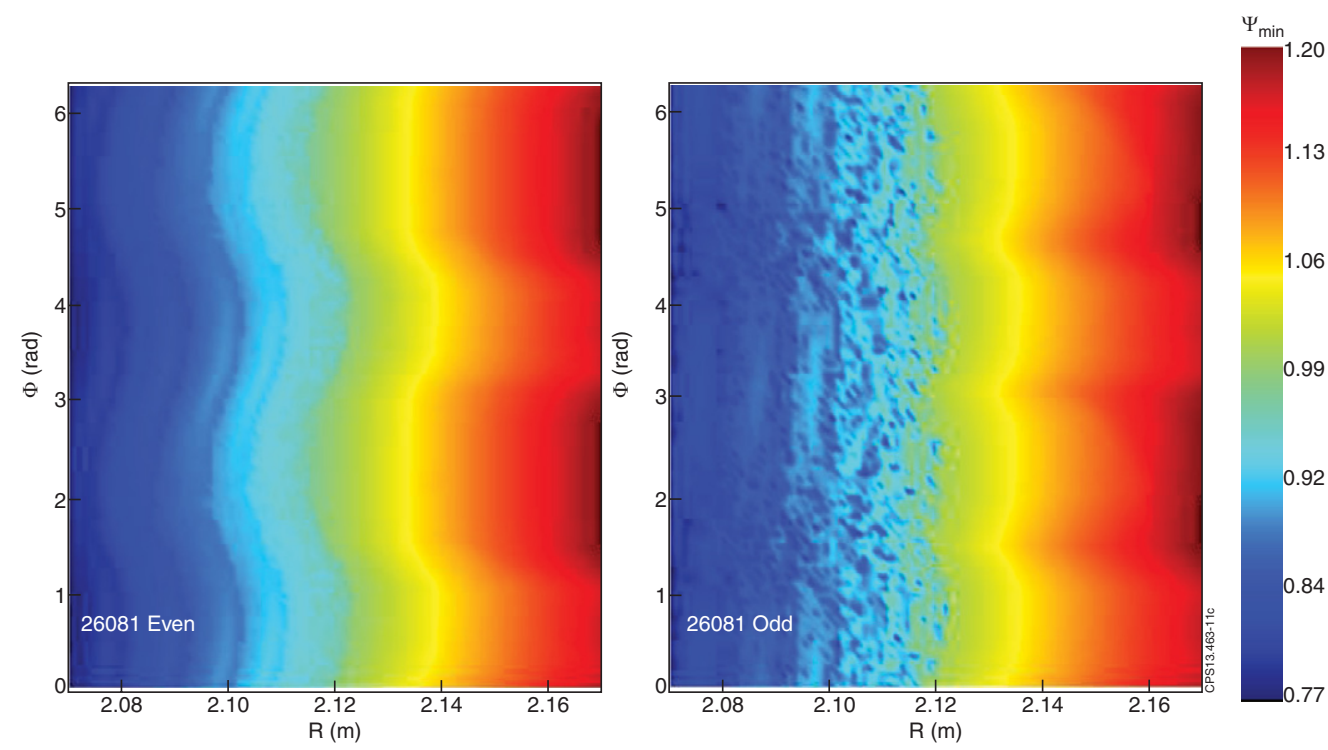

Figure 11. ASDEX Upgrade. The plasma boundary predicted by the ERGOS code as a function of toroidal angle for ASDEX Upgrade shot 26081 when either (left) even parity or (right) odd parity $n=2$ non-axisymmetric fields are applied.

surface, normalized to the major radius (see p 47 of [38]):

$$
b_{\text {res }}^{\mathrm{r}}=\frac{2\left|b_{m, n}^{1}\right|}{R_{0}\left\langle\nabla \sqrt{\psi_{\mathrm{pol}}}\right\rangle} .
$$

It should be noted that this database contains displacements measured by different diagnostics, at different locations, in plasmas with different collisionalities and shapes, and with both static and rotating RMPs of different spectra applied, so at best it can provide qualitative trends. Figure 9 shows a linear correlation between the measured 3D displacement (here averaged between all toroidal positions with highly spatially resolved data, removing of course the expected null points) and the resonant field as defined in equation (1). This implies that, whilst vacuum modelling cannot give an accurate determination of the amplitude of the 3D displacement, it can provide a qualitative prediction on the variation of the $3 \mathrm{D}$ corrugation with respect to the configuration of the applied non-axisymmetric fields. Finally, it is worth noting that there is a very weak correlation between the measured displacements and the normalized pressure in this database. Although this suggests that plasma response is not a leading order parameter in determining the displacements across this multi-machine database, it should be noted that a more appropriate parameter would be $\beta_{\mathrm{N}} / \beta_{\mathrm{N}}^{\text {no-wall }}$, since this might be expected to determine the expected plasma response more than the absolute pressure.

At the resonant field expected in ITER, this linear fit would give an empirical scaling for the displacement in ITER of $\pm 2.25 \%$ of the minor radius, equating to $9 \mathrm{~cm}$ peak-to-peak. This means that if the plasma control system were to exacerbate the displacement and apply an $n=0$ correction to the $n=3$ or $n=4$ corrugation, the displacement of the boundary could decrease the coupled ICRH power for the antenna affected by a factor of 2 for steep far SOL profiles and increase the power fluxes to the ITER first wall components although the increased power fluxes would still be within acceptable limits. This is far from a robust prediction, but nonetheless, exemplifies why this could be a concern for ITER operation.

\section{Modelling of displacements due to RMPs in present machines}

There are two distinct effects that might lead to the plasma boundary displacements that are measured in multiple devices: displacement of the internal flux surfaces due to the kink response, and displacement of the stable and unstable manifolds which form the axisymmetric separatrix. The kink response, which arises due to external drive of the least stable mode by the applied RMP, intrinsically involves the plasma response, and cannot be modelled with a vacuum code. In contrast, the manifold displacements cannot be modelled by a code, such as an ideal three-dimensional equilibrium code (e.g. VMEC), which does not include the separatrix and $\mathrm{X}$-point in the computational domain. However, the third class of codes used here, resistive MHD codes which include the X-point, can model both the plasma response on closed flux surfaces and the manifold displacements. The displacements observed in present-day machines presented in section 2 have been modelled using a range of numerical modelling tools-(1) vacuum field line tracing codes giving the change in the position of the magnetic boundary, which is represented by the difference in position of either stable and unstable manifolds when RMPs are applied; (2) ideal three-dimensional equilibrium codes, which do not solve the Grad-Shafranov equation, but instead perform an energyminimization procedure to find an equilibrium state, though the codes used in this study assume ideal, nested flux surfaces; (3) resistive MHD codes, which can capture the screening of the applied field, the response of the plasma, the effects of plasma rotation and an X-point geometry. All these models, with very different approaches and different approximations, have been compared to machine data in different parameter space with varying levels of success, illustrating that extrapolation of the 3D corrugation likely in ITER when RMPs are applied is a complex problem. 


\subsection{Vacuum field line tracing}

The simplest prediction for a boundary corrugation comes from vacuum field line tracing, ignoring the plasma response and the shielding of the applied field, which have been shown to be important for understanding ELM behaviour in the presence of RMPs. At the same time, vacuum modelling has also given a reliable prediction for strike-point splitting on the divertor plates, indicating that it can be reliable in the scrape-off layer and right at the plasma edge at least. To some extent, the applicability of vacuum modelling is also confirmed by figure 9 which shows a strong correlation between the resonant field given by vacuum modelling and the measured corrugation of the plasma boundary across five different machines. Figure 10 shows a laminar plot illustrating the connection length of field lines from a vacuum field line tracing calculation with an $n=2$ RMP applied in ASDEX Upgrade [25]. The dashed black line is an effective plasma boundary, marking the envelope of the stable and unstable manifolds, which can be compared to the solid black line marking the position of the axisymmetric separatrix. There is a toroidal corrugation, though it is only $\pm 3 \mathrm{~mm}$, which agrees well with the sub-cm boundary displacement observed experimentally, as seen in figure 6 .

In order to confirm this prediction, another independent vacuum field line calculation for ASDEX Upgrade has been performed with the ERGOS code [38]. Figure 11 shows the plasma boundary prediction in a laminar plot produced by ERGOS when either even parity or odd parity $n=2$ RMPs are applied. For this equilibrium, the even parity case is well aligned with the equilibrium q-profile, as evidenced by the larger corrugation amplitude, though in both cases the displacements are predicted to be sub-cm, in line with figures 10 and 6 .

However, there are examples where vacuum modelling somewhat under predicts the displacements seen experimentally. For instance, the displacements seen when rotating $n=2$ RMPs are applied in DIII-D are compared to predictions from the TRIP3D-MAFOT vacuum magnetic field model [59] in figure 12 [29]. The vacuum modelling predicts only $\pm 2.5 \mathrm{~mm}$ displacement whereas the BES measurements give $\pm 0.8-1.2 \mathrm{~cm}$, representing a factor of 4-5 times larger than the vacuum prediction. Whilst the vacuum prediction does not include any screening effects, it also neglects the amplification from the plasma response, which seems to be important in this case.

A similar under prediction of the boundary corrugation is given by vacuum modelling of MAST discharges with an $n=3 \mathrm{RMP}$ applied. Figure 13 shows a laminar plot from ERGOS vacuum modelling from which the vacuum prediction of the displacement can be inferred. The experimental measurements show a boundary shift of $\pm 2.5 \mathrm{~cm}$ [27], which is a factor of four larger than the vacuum prediction of $\pm 5-6 \mathrm{~mm}$ shown in figure 13 .

Whilst vacuum modelling predicts the edge displacement in ASDEX Upgrade accurately, it cannot explain the larger displacements observed in MAST or in some cases in DIII-D. In order to compare these cases directly, the resonant field in MAST and ASDEX Upgrade is compared using the same vacuum field line tracing code, ERGOS, as shown in figure 14. It is evident that the application of $n=6$ RMPs in MAST leads to a factor of five larger local resonant field than the $n=2$ field applied in ASDEX Upgrade. This larger resonant field
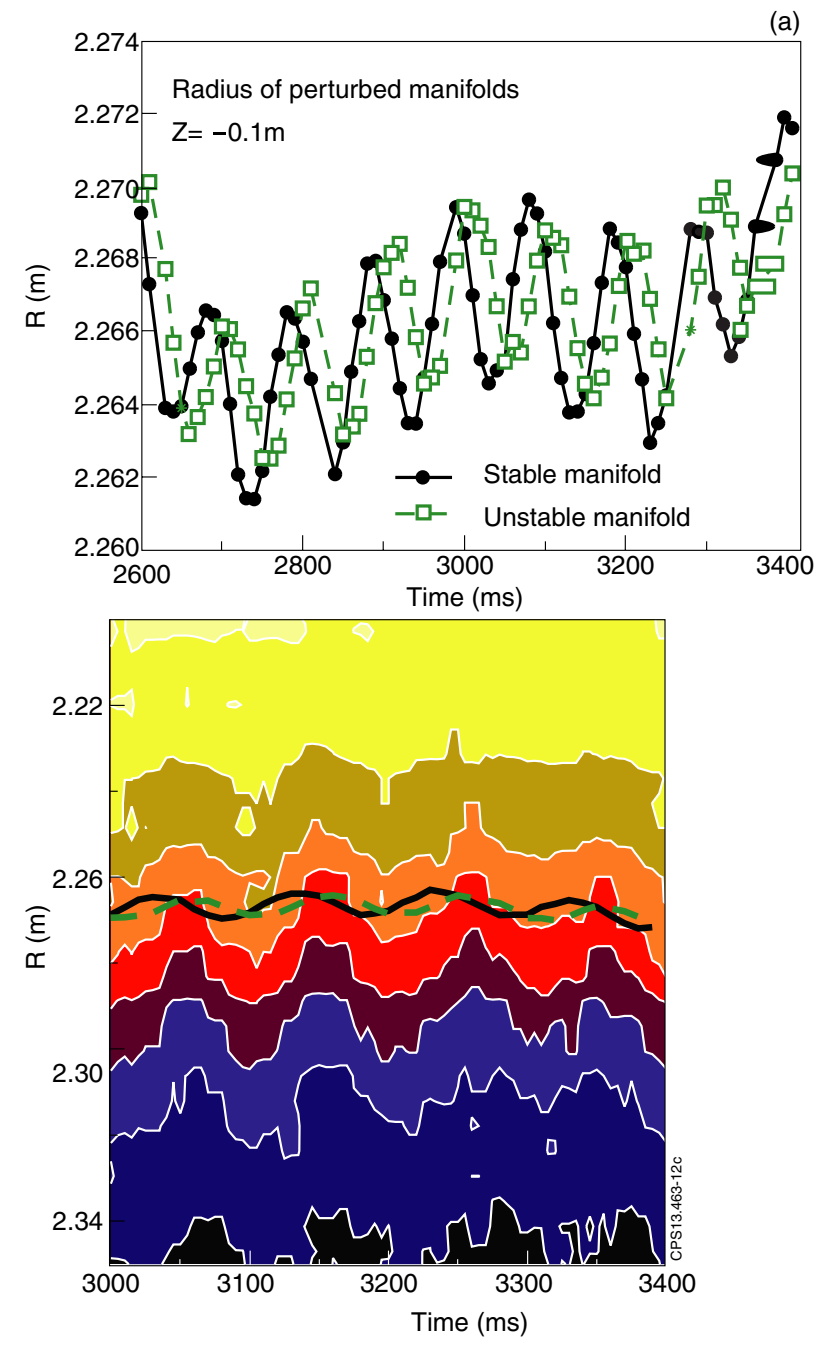

Figure 12. DIII-D. (a) Temporal evolution of the major radius $R$ of the (filled black circles and solid black line) stable and (open green squares and green dashed line) unstable manifolds at an elevation of $z=-0.1 \mathrm{~m}$ on the LFS midplane computed by the

TRIP3D-MAFOT vacuum magnetic field model (no plasma response). Each manifold is displaced by $4-5 \mathrm{~mm}$ over a cycle of the $n=2$ RMP rotation. (b) Colour contour plot of the beam emission intensity at an elevation $z=-0.1 \mathrm{~m}$ versus $R$ and time in the LFS boundary, showing the displacement in the beam emission profile. The measured BES displacement is compared with the major radius $R$ of the (black solid line) stable and (green dashed line) unstable manifolds at an elevation of $z=-0.1 \mathrm{~m}$ that result from the splitting of the divertor separatrix due to the non-resonant interaction with the $n=2$ magnetic perturbation. The BES displacement of $1.8-2.4 \mathrm{~cm}$ over 18 cycles of the $n=2$ rotation is 4-5 times the predicted vacuum magnetic field model displacement. Reproduced with permission from Moyer R.A. et al $2012 \mathrm{Nucl}$. Fusion 52 123019. Copyright 2012 IAEA Vienna.

partially explains why the MAST plasmas experience larger edge corrugations, though the underprediction afforded by vacuum modelling in figure 13 suggests that there is a plasma amplification of the applied field as well.

\subsection{Ideal three-dimensional equilibrium modelling}

The next level of complexity in modelling 3D displacements is ideal $3 \mathrm{D}$ equilibrium modelling. It should be noted that 
(a)

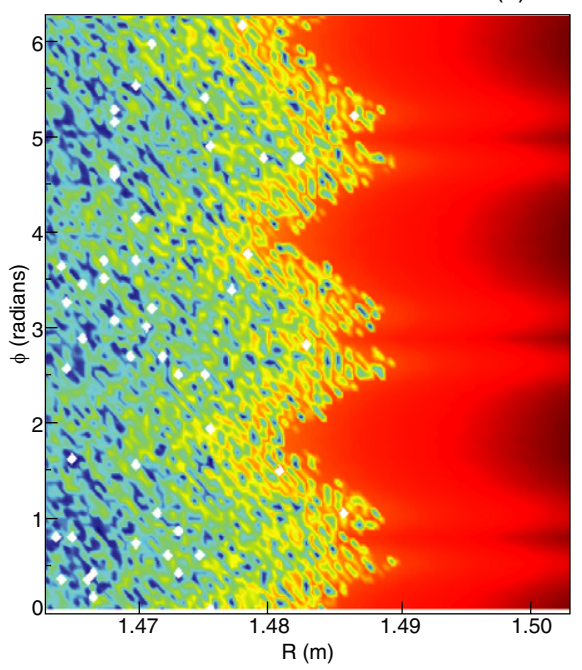

(b)

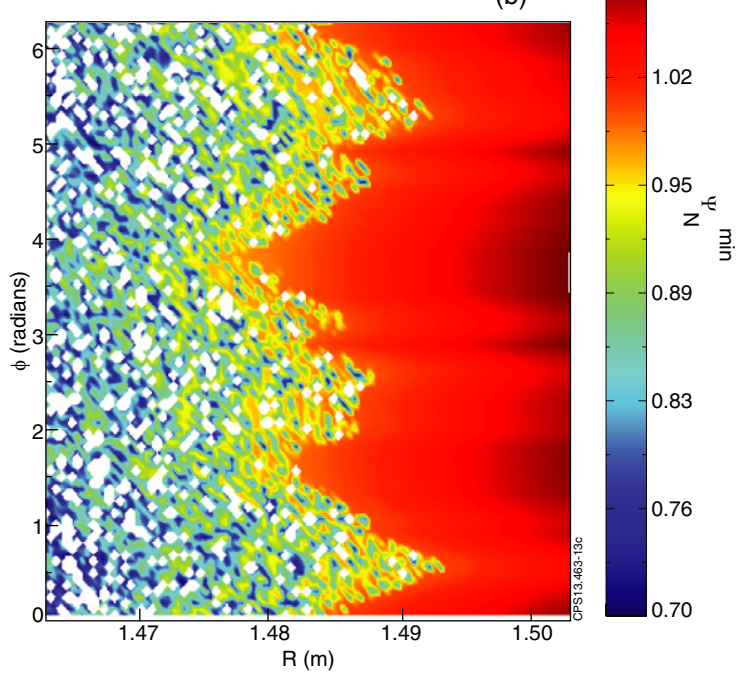

Figure 13. MAST. A laminar plot generated by the ERGOS code showing the minimum normalized flux experienced by each field-line traced from each given poloidal position in one toroidal plane. The field-lines are followed for 200 toroidal turns or until they reach the divertor target. The deformation of the 'boundary' in MAST when an $n=3(a)$ even parity and $(b)$ odd parity RMP is applied predicted by ERGOS is $\pm 5-6 \mathrm{~mm}$, which is significantly less than the displacement measured experimentally [27], where a displacement of $\pm 2.5 \mathrm{~cm}$ is observed when the RMPs are switched on.

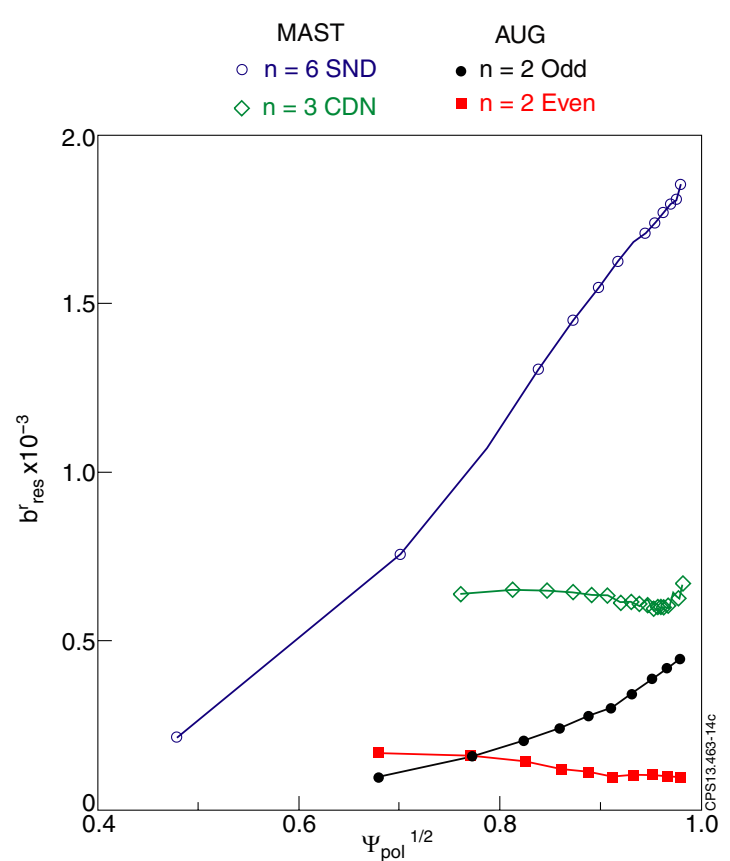

Figure 14. ASDEX Upgrade and MAST. The resonant component of the applied magnetic field as a function of the minor radius as predicted by the ERGOS vacuum field line following code for a MAST connected double null plasma with an $n=3$ RMP applied and a MAST singlt null plamsa with an $n=6$ RMP applied compared to ASDEX Upgrade plasma with $n=2$ field applied with either odd or even parity.

3D equilibrium codes used here do not handle an X-point geometry, and consequently cannot calculate the manifold displacements due to the RMP. Displacements of the plasma boundary equating to less than $1 \%$ of the minor radius observed experimentally are also replicated using such 3D equilibrium modelling in both ASDEX Upgrade and in NSTX. Figure 15

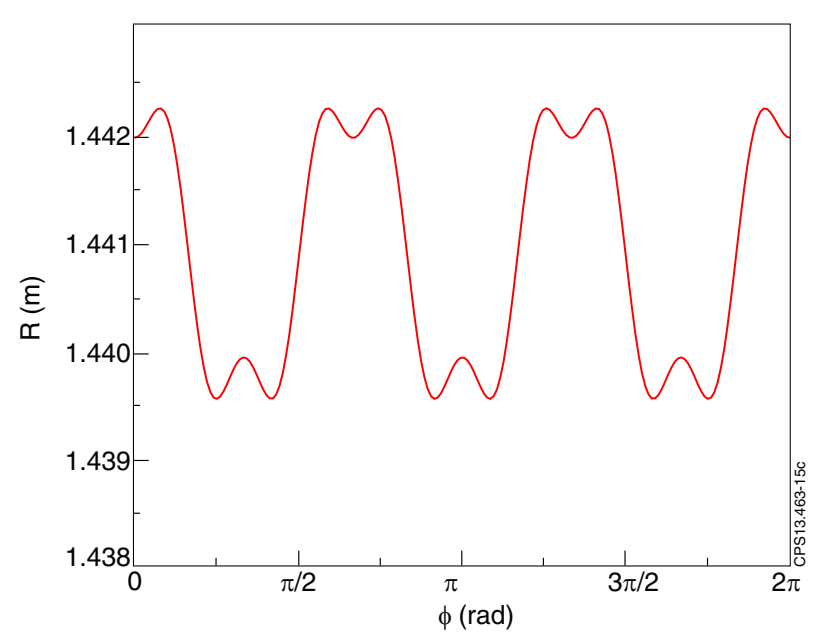

Figure 15. NSTX. The toroidal dependence of the radial position of the plasma boundary as predicted by VMEC when an $n=3$ RMP is applied in NSTX. There is an $n=12$ toroidal field ripple in addition to the non-axisymmetric $n=3$ structure.

shows the toroidal dependence of the position of the plasma boundary predicted by the VMEC 3D equilibrium code [39] when an $n=3 \mathrm{RMP}$ is applied in NSTX [40]. It is evident that the boundary is expected to be perturbed by only $\pm 1 \mathrm{~mm}$, in good agreement with the measured invariance of the pedestal foot position seen in figure 8 .

However, in contrast to vacuum modelling, threedimensional ideal equilibrium calculations also give good agreement with experimental cases where larger boundary perturbations are observed. Figure 16 shows STELLOPT predictions for the plasma boundary displacement as a function of toroidal and poloidal angles in a DIII-D plasma when a static $n=3 \mathrm{RMP}$ is applied to an up/down symmetric double null plasma [45]. The STELLOPT code [46] is designed to 


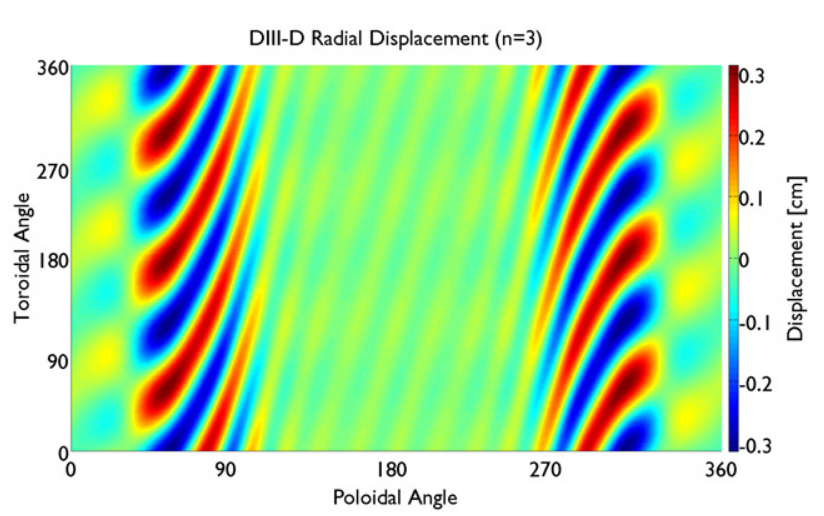

Figure 16. DIII-D. The plasma boundary displacement as a function of toroidal and poloidal angles as predicted by the STELLOPT code for a DIII-D plasma with an $n=3$ RMP applied. Adapted from Lazerson S.A. et al 2014 Three dimensional equilibrium reconstruction on the DIII-D device $\mathrm{Nucl}$. Fusion submitted.

optimize the VMEC 3D MHD equilibrium to a set of target physics parameters derived from different plasma diagnostics. The simulation predicts a boundary corrugation of $\pm 3 \mathrm{~mm}$, maximized $45^{\circ}$ above and below the outer midplane [48].

Ideal 3D equilibrium modelling has also been applied for MAST plasmas when RMPs are applied. Figure 17 shows the radial position of the last closed flux surface at the outboard midplane as predicted by VMEC when an $n=3$ RMP is applied to a connected double-null MAST plasma where ELM mitigation is observed [13]. Without an applied $n=3$ field, there is a natural $n=12$ boundary corrugation associated with the toroidal field ripple, though this is only $\pm 2 \mathrm{~mm}$, well below the resolution of any diagnostic. However, when an $n=3$ field is applied, there is a clear edge corrugation of $\pm 7 \mathrm{~mm}$, which is maximized at the midplane.

This can be compared to the edge corrugation predicted by VMEC when an $n=6$ RMP is applied to a connected double null MAST plasma, as shown in figure 18. It is evident that the boundary corrugation resultant from the $n=6$ RMPs is strongly dependent on the alignment of the applied field with the equilibrium field. In the case of the even parity field, there is negligible distortion, whereas the odd parity $n=6 \mathrm{RMP}$ gives rise to a $\pm 1 \mathrm{~cm}$ corrugation.

When the applied field is optimally aligned with the plasma field - at which point the ELM mitigation is maximized [13] - the corrugation is even larger. Figure 19 shows ANIMEC modelling of a MAST connected double null plasma when an $n=3$ field is applied with a pitch angle to align the field with the $q$-profile. In this case the edge corrugation is $\pm 2.5 \mathrm{~cm}$, equating to $\pm 4.5 \%$ of the minor radius. Also shown in figure 19 are data from different diagnostics in MAST when this phase of $n=3$ RMP is applied, showing reasonable agreement between the measured position of the plasma boundary and the predicted edge position from ANIMEC, suggesting that ideal 3D MHD equilibrium modelling is able to replicate the experimental shifts observed in MAST accurately.

However, there are examples where ideal 3D equilibrium modelling does not replicate the large boundary corrugations observed in some experiments. For instance, figure 20 shows a VMEC 3D equilibrium for a JET plasma with $80 \mathrm{kAt}$ in the external coils producing an $n=2$ field. This $n=2 \mathrm{RMP}$ is observed to produce a boundary displacement of $\pm 3 \mathrm{~cm}$ [34], whereas VMEC predicts only a $\pm 1 \mathrm{~cm}$ boundary corrugation for this plasma equilibrium.

\subsection{Resistive $M H D$ modelling}

The next level of complexity is to use a resistive MHD model which includes the plasma response to the applied field, allowing amplification by marginally stable MHD modes as well as screening of the field [47]. Figure 21 shows such a comparison between the electron temperature pedestal measured by the Thomson scattering diagnostic in DIII-D and that predicted by the M3D-C ${ }^{1}$ MHD code [44]. In this case, the displacements observed experimentally are reasonably modelled by linear resistive MHD. It should be noted that the modelling does not include intrinsic error fields.

Furthermore, M3D-C $\mathrm{C}^{1}$ is also able to replicate the dependence of the corrugation amplitude on plasma parameters. In DIII-D, larger displacements are observed at larger $q_{95}$ : plasmas with $q_{95}=3.1$ have $\xi_{a} \approx 4 \mathrm{~mm}$ whereas plasmas at $q_{95}=3.8$ have $\xi_{a} \approx 8 \mathrm{~mm}$. Such a dependence on plasma current is replicated by M3D-C ${ }^{1}$ as shown in figure 22 . This trend follows the vacuum field intuition, since $\delta B / B$ (via $I_{\mathrm{RMP}} / I_{\mathrm{P}}$ ) increases with $q_{95}$, here changed by scaling the current. Indeed, vacuum modelling based on these shots recovers this trend [49]. It is contrary, however, to the intuition one might apply if plasma amplification plays a key role in determining the edge displacement. One might assume that higher current sustains higher pressure gradient, making edge modes more unstable, hence amplifying the applied field and leading to larger displacements, rather than the smaller edge corrugation observed. However, resistive MHD including rotation is able to capture both amplification and screening and as such includes the pertinent physics to allow good agreement across a wide range of plasma conditions.

The simulations in figures 21 and 22 use a linear MHD approximation. Here we consider linear modelling as appropriate provided $\left|\mathrm{d} \xi_{r} / \mathrm{d} r\right|<1$. If this condition is not met, then the flux surface displacements overlap, implying a breakdown of the condition $B \cdot \nabla T_{\mathrm{e}}=0$. In order to verify the applicability of the linear model in these conditions, the displacements predicted using linear MHD have been compared to non-linear simulations, as shown in figure 23. The overlap criterion that $\left|\mathrm{d} \xi_{r} / \mathrm{d} r\right|<1$ is clearly met across the whole minor radius, and commensurately, the boundary displacements, and indeed the whole pedestal electron temperature profile, is in good agreement in the linear and nonlinear simulations.

Another nonlinear resistive MHD code, JOREK [50], has been used to simulate MAST [70] and JET plasmas [43] when RMPs are applied. JOREK simulations with realistic resistivity and flows have been performed for JET discharges with an $n=2$ RMP applied. For discharge 77329, the measured displacement resulting from the application of the $n=2 \mathrm{RMP}$ is $\pm 1 \mathrm{~cm}$. Figure 24 shows the predicted displacements at the plasma top, X-point and midplane respectively when JOREK is run without realistic diamagnetic and toroidal flows included. In this case the boundary displacement is $\pm 1.2 \mathrm{~cm}$. When realistic flows are included in the simulation, the boundary displacement is halved, to $\pm 6 \mathrm{~mm}$, as shown in figure 25 . 

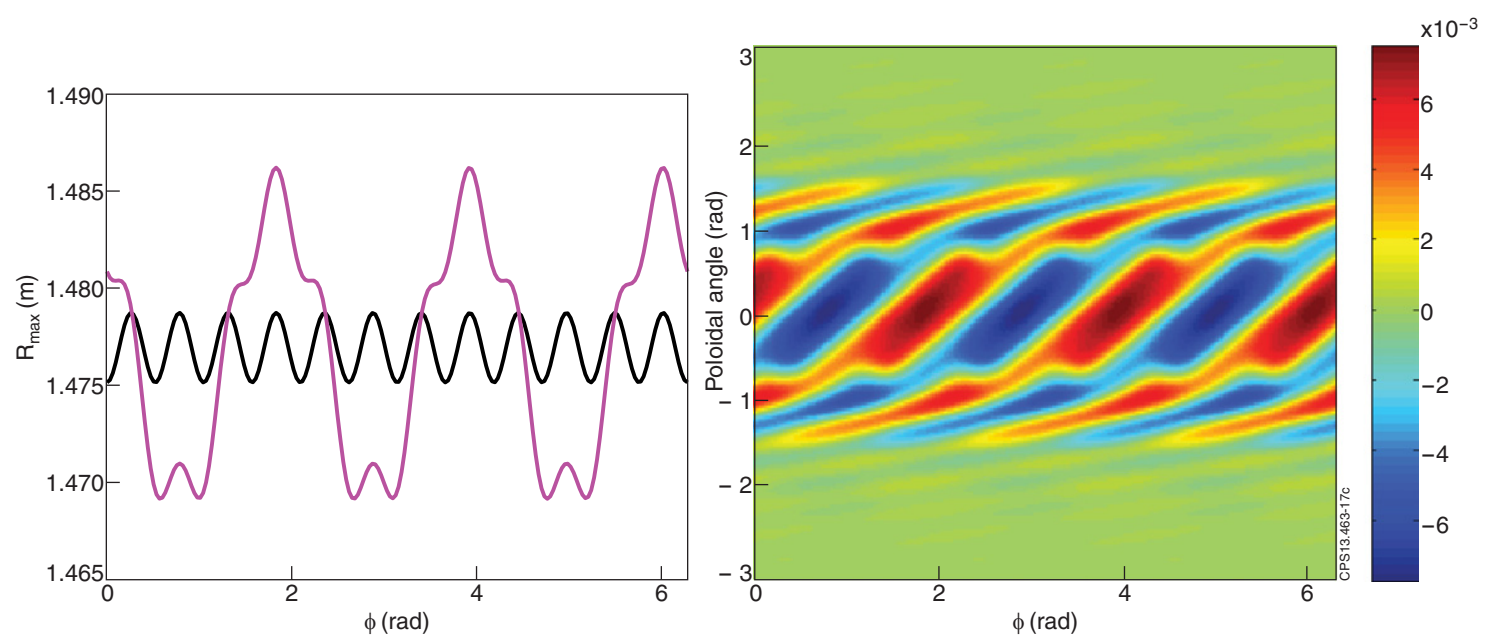

Figure 17. MAST. (left) The plasma boundary predicted by VMEC for a MAST connected double null plasma when an $n=3$ RMP is applied (pink) compared to the case with no RMP but with toroidal field ripple (black). (right) The boundary displacement predicted by VMEC as a function of toroidal and poloidal angle, showing maximum displacement on the midplane for this double-null plasma.
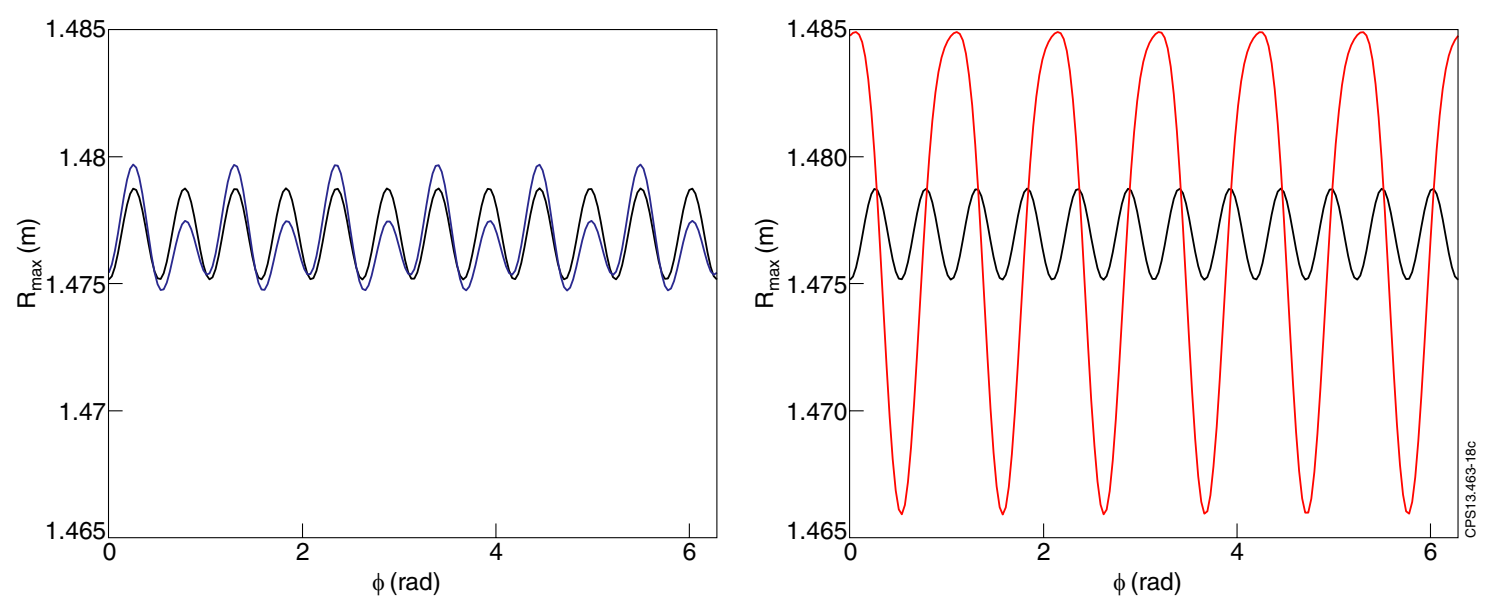

Figure 18. MAST. The plasma boundary predicted by VMEC for a MAST connected double null plasma when an $n=6$ RMP is applied compared to the case with no RMP but with toroidal field ripple when the RMP has (left) even parity configuration and (right) an odd parity configuration.

\subsection{Comparison of different numerical models}

In section 2 the range of measured displacements in presentday machines was detailed, ranging from $\pm 3.5 \%$ of the minor radius to negligible edge corrugation. In a similar vein, the models needed to replicate the experimental observations range from simple vacuum field line tracing, to ideal 3D equilibria and nonlinear resistive MHD simulations. Whilst there is a strong correlation between the resonant field predicted by vacuum models and the measured displacement, seen in figure 9, vacuum modelling does not always accurately replicate the absolute magnitude of the empirical observations, even if it does provide qualitative trends with respect to the plasma parameters. Indeed, in all cases where large displacements are observed, either ideal 3D equilibrium modelling (in MAST) or resistive MHD including plasma amplification effects (in DIII-D and JET) are required to replicate the observations. However, there remains uncertainty in all of these models, in the level of plasma and rotation screening of the applied fields, in the damping caused by the applied field and in the nonlinear interaction of 3D fields, marginally stable modes and rotation. Therefore it is prudent to use all models available and take the worst case scenario when making predictions for ITER, as follows in section 4.

\section{Modelling of displacements due to RMPs in ITER}

As in section 3, the displacements expected in ITER are modelled using vacuum field line tracing, ideal 3D equilibrium simulation and nonlinear resistive MHD codes. The coil set used in these simulations is shown in figure 26, including the latest design for the in-vessel control coils (IVCCs) [51] planned for ITER. The in-vessel control coils for ITER were specified upon the basis of vacuum modelling [52,53] which stipulated the necessary fields such that the RMP-induced islands overlapped across a certain width of the minor radius which was found to result in ELM suppression in DIII-D experiments [55]. The present ITER design with a set of 27 in-vessel six-turn coils (above, below and at the midplane, as shown in figure 26) permits a maximum current carrying 


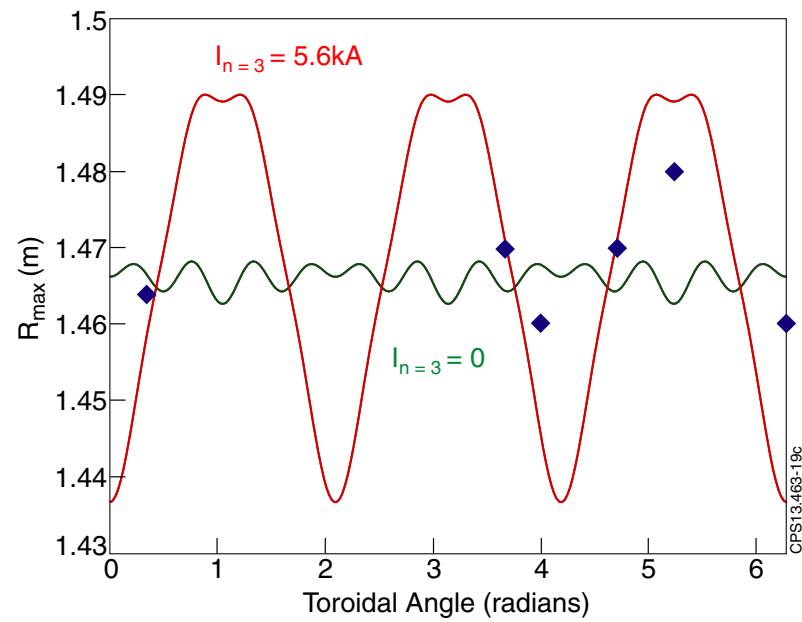

Figure 19. MAST. The plasma boundary displacement as a function of toroidal angle as predicted by the ANIMEC code compared to measurements of the midplane displacement in MAST when an $n=3 \mathrm{RMP}$ is applied.

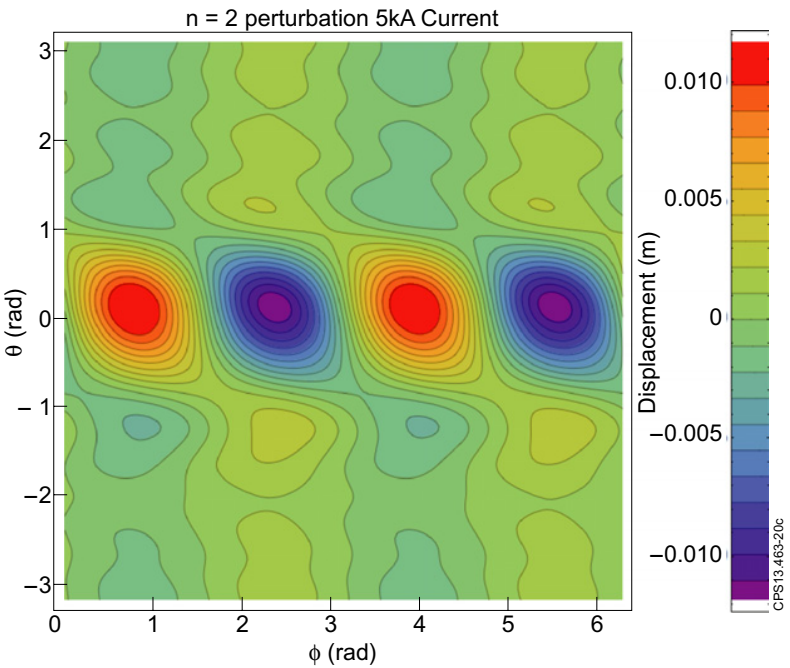

Figure 20. JET. The plasma boundary displacement as a function of toroidal and poloidal angles as predicted by the VMEC code when an $n=2$ RMP is applied in JET.

capability of $90 \mathrm{kA}$-turns. Vacuum modelling suggests that current in the IVCC in excess of $50 \mathrm{kAt}$ (for $n=3$ ) and $70 \mathrm{kAt}$ (for $n=4$ ) should be sufficient to meet the empirical criteria established for ELM suppression [56]. However, a solid physical basis for the magnitude and structure of the nonaxisymmetric fields which will avoid type-I ELMs remains to be developed. Indeed, it has been suggested that ELM control occurs in part because of the 3D corrugation of the plasma boundary [21]. In this study we consider the edge distortion associated with half the possible 3D field (the marginal level predicted for ELM suppression) as well as full field.

\subsection{Vacuum field line tracing}

The TRIP3D-MAFOT vacuum field line tracing code [57] has been used to predict the boundary displacement expected in a 15 MA baseline scenario when full current is applied in the IVCCs in an $n=3$ configuration. Plasma simulations that do

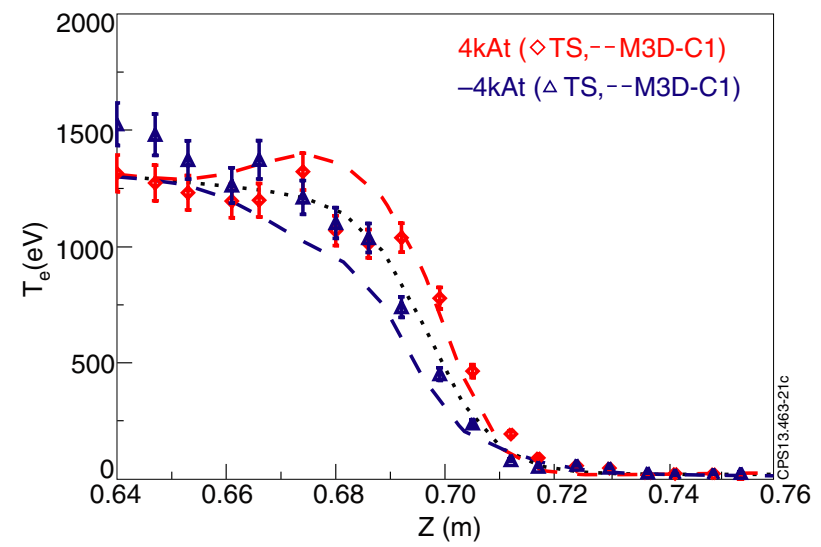

Figure 21. DIII-D. The electron temperature profile as a function of $Z$ as measured by the Thomson scattering diagnostic in DIII-D (symbols) compared to the prediction from the M3D-C $\mathrm{C}^{1}$ linear MHD code for two phases of an applied $n=3$ RMP, showing good agreement and a clear edge displacement. Reproduced with permission from Ferraro N.M. et al 2013 Nucl. Fusion 53073042. Copyright 2013 IAEA Vienna.

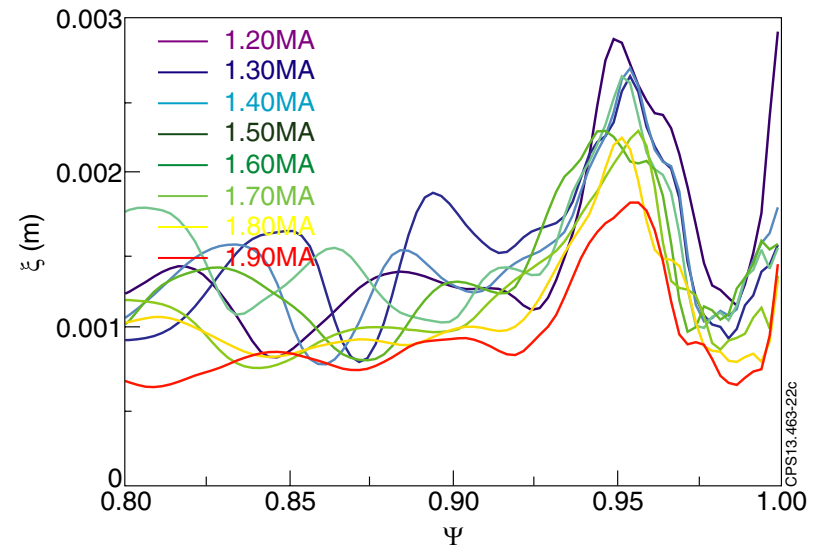

Figure 22. DIII-D. The flux surface perturbation as a function of radius for different values of plasma current with an applied $n=3$ RMP, as modelled by linear M3D-C ${ }^{1}$ runs. It is clear that the edge displacement increases for lower $I_{\mathrm{p}}$, that is to say for higher $q_{95}$ in this case.

not include the X-point geometry typically take an isotherm or isobar as a proxy for the boundary position. TRIP3DMAFOT includes the X-point geometry, and calculates the positions of the stable and unstable manifolds that result from the splitting of the separatrix by non-axisymmetric magnetic perturbations $[58,59]$. These homoclinic tangles are computed to be particularly complex and extended near the X-point [60]. This concept of lobe structures formed by the invariant manifolds of the perturbed field has been used to explain the splitting of the divertor leg footprints observed on strike-point targets during RMP experiments [58, 61-64], suggesting that at least for the boundary and scrape-off layer, vacuum modelling gives a good description of the effect of the applied fields. Furthermore, vacuum modelling with an appropriate screening model has been used to get a good correspondence between the X-point lobe strcutures measured in MAST $[12,71]$. It is shown in reference [49] that the boundary displacement at the outer midplane in ITER is predicted by the vacuum modelling to be $\pm 3 \mathrm{~cm}$ as defined by the movement of the 

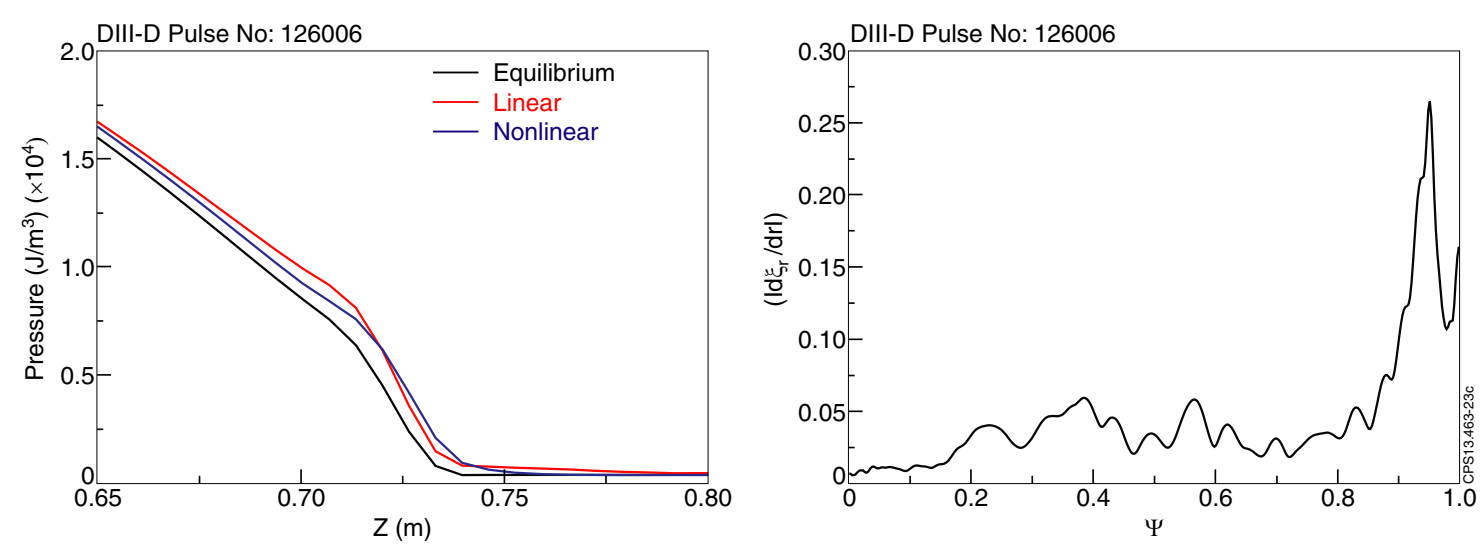

Figure 23. Left: the plasma electron pressure as a function of vertical position (along the line of sight of the Thomson scattering) in DIII-D as predicted by the M3D-C ${ }^{1}$ code, comparing the results of linear and non-linear simulation when an $n=3$ RMP is applied. Right: linear modelling is assumed to be appropriate when $\left|\mathrm{d} \xi_{\mathrm{r}} / \mathrm{d} r\right|<1$ [44] and this condition is met across the minor radius for these DIII-D plasmas.

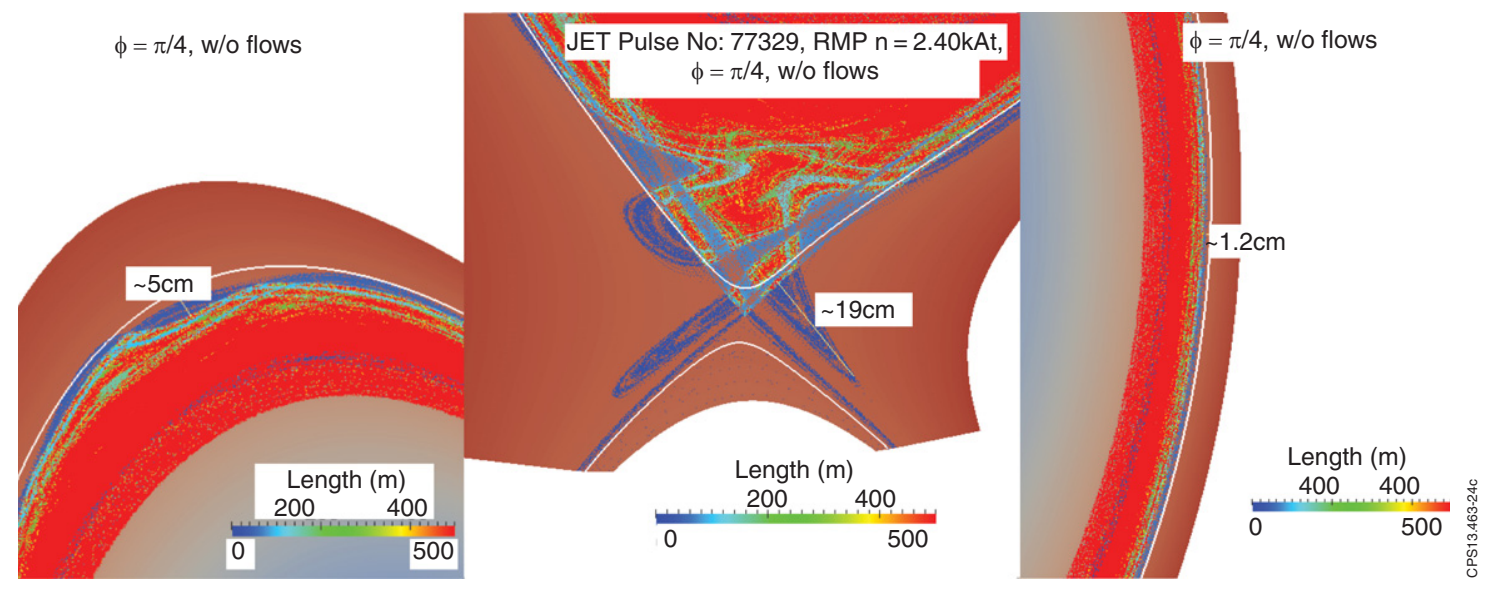

Figure 24. JET. Poincare plots showing the (left) top, (middle) lower X-point region and (right) midplane of JET discharge 77329 modelled using JOREK without realistic diamagnetic and toroidal flows included.
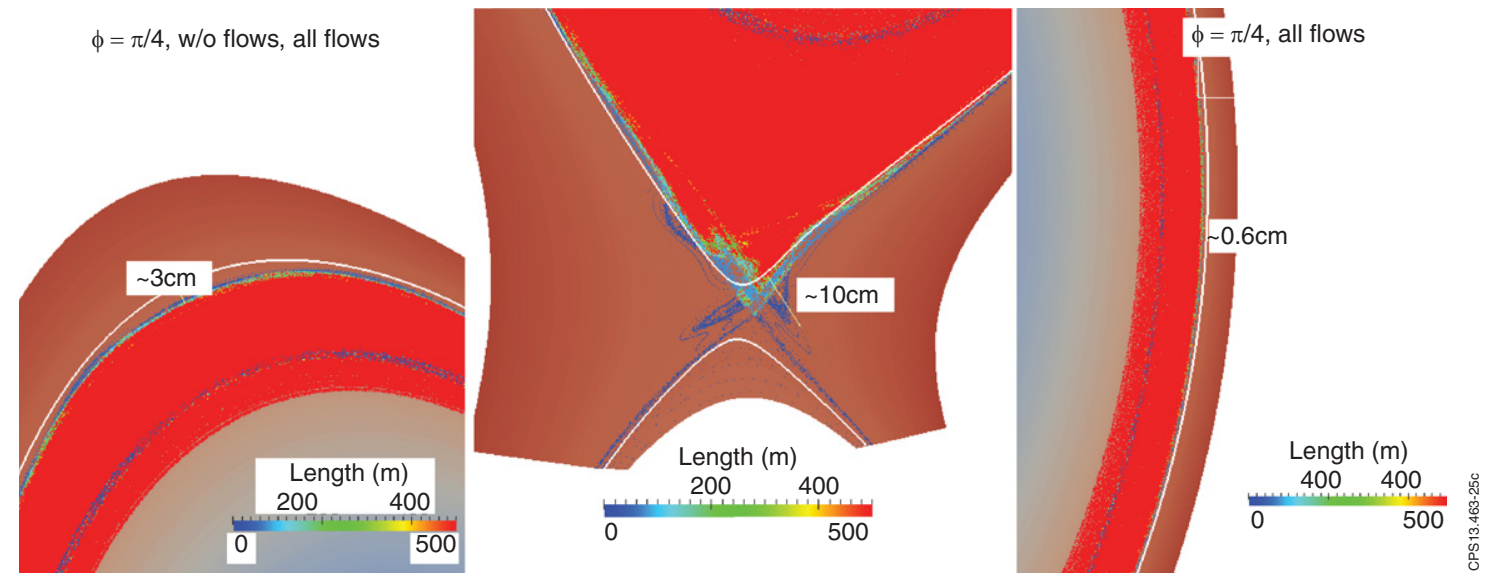

Figure 25. JET. Poincare plots showing the (left) top, (middle) lower X-point region and (right) midplane of JET discharge 77329 modelled using JOREK when realistic diamagnetic and toroidal flows are included.

unstable manifold that has the largest range of movement at this toroidal location. This represents $\pm 1.5 \%$ of the minor radius in ITER.

It is possible to use a simple analytic model to find the dependence of the boundary displacement on the equilibrium $q$-profile, and thus on the resonance of the applied field.
We start by taking the 3D magnetic perturbation spectrum from an M3D-C ${ }^{1}$ response calculation for the baseline ITER scenario with a $3.8 \mathrm{keV}$ pedestal temperature and $22.5 \mathrm{kAt}$ in the midplane-IVCCs. The outermost radial point in the domain is chosen, at $\psi_{N}=0.9988$ and $q=4.262$. Three-dimensional local equilibrium theory is used to study the sensitivity of the 


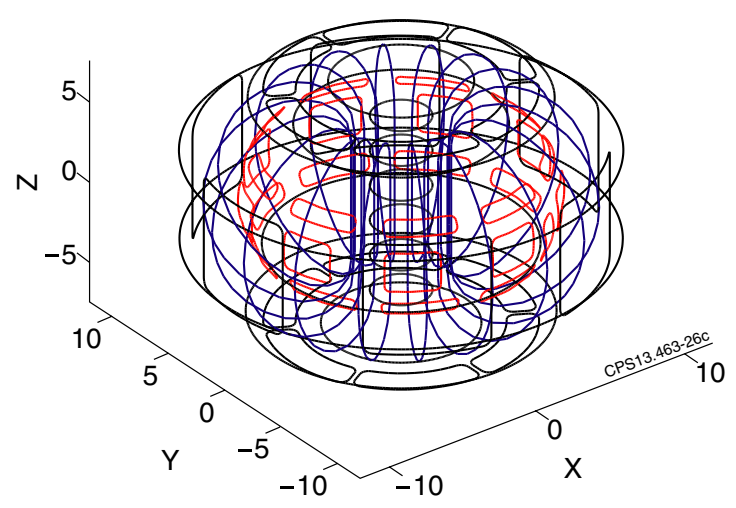

Figure 26. The coil set used in ITER simulations using VMEC, with the in-vessel control coils marked in red.

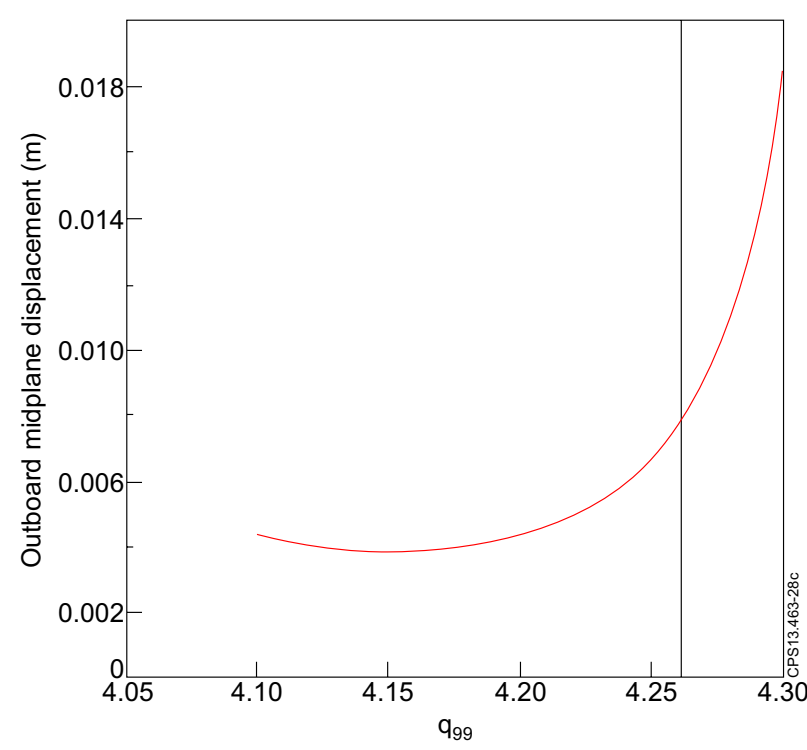

Figure 27. ITER. The edge displacement as a function of the edge safety factor found from simple 3D local equilibrium theory.

3D deformation size at this surface to the local value of the safety factor. Starting with the axisymmetric flux surface shape (taken from the initial 2D equilibrium used in the M3D-C ${ }^{1}$ response calculation), we add a spectrum of $3 \mathrm{D}$ flux surface deformations which match the $3 \mathrm{D}$ radial magnetic perturbation spectrum from M3D-C ${ }^{1}$. This approach follows the procedure described in [41]. In the high aspect ratio, circular cross section limit the relationship between a deformation of a given helicity $(\gamma)$ and the radial magnetic perturbation with which it is associated is given by $B_{r} / B_{0}(m, n)=\left(\gamma / R_{0}\right)(n q-(m-1))$, where an $m=4$ deformation is associated with an $m=3$ radial magnetic perturbation. With the $q$ value taken from the response calculation we find a $3 \mathrm{D}$ flux surface displacement at the outboard midplane of $\pm 7 \mathrm{~mm}$. We then repeat the calculation of the flux surface displacement with varying safety factor, but holding the 3D perturbation spectrum fixed. The energy needed to bend equilibrium magnetic field lines decreases as low order rational surfaces are approached, and consequently the displacement size has a $1 / x$-type sensitivity where $x$ is the distance to the nearest low order rational surface. The validity of these calculations comes into question as the rational surface is approached, as the resonant component of the radial magnetic perturbation may be suppressed by flow screening, and in principle new radial perturbation spectra should be used. Here we limit our calculations to a range of $q$ values where the radial magnetic perturbation spectra used should remain valid. These calculations suggest that the displacement of the last closed flux surface will have some sensitivity to the $q$ value, as seen clearly in figure 27. Small changes in the edge $q$ value may be able to significantly modify the displacement of the last closed flux surface.

\subsection{Ideal three-dimensional equilibrium modelling}

However, a plasma response to the applied fields is expected in ITER [65]. 3D equilibrium simulation of the various ITER scenarios with either $n=3$ or $n=4$ RMPs applied has been performed using the VMEC code. The profiles for the baseline scenario are taken from the latest transport simulations of ITER 15 MA plasmas using the CORSICA code [66]. Linear pedestal stability analyses based upon these equilibria have found that finite- $n$ peeling-ballooning modes are unstable for temperature pedestals such that $T_{\mathrm{e} \text {,ped }}>5.9 \mathrm{keV}$ [68].

The NEMEC code [39] (a free boundary version of VMEC, which for the purposes of this study is ostensibly identical and checked against VMEC [69]) has been used to assess the boundary displacements in various ITER scenarios with both $n=3$ and $n=4$ applied RMPs at different field strengths. The simulations naturally include TF ripple effects (without ferritic inserts), which alone can induce corrugations of $\pm 7-8 \mathrm{~mm}$. Four current distributions in the IVCCs have been studied, namely an $n=4$ configuration with $I_{\mathrm{IVCC}}=90 \mathrm{kAt}$ and $I_{\mathrm{IVCC}}=30 \mathrm{kAt}$ and an $n=3$ configuration with $I_{\mathrm{IVCC}}=90 \mathrm{kAt}$ and $I_{\mathrm{IVCC}}=45 \mathrm{kAt}$ [67]. The $n=4$ cases show a strong addition of $n=5$, whilst the $n=3$ cases have additional $n=6$ amplitudes. In all twelve NEMEC calculations (two coil configurations $\times$ two RMP amplitudes $\times$ three plasma scenarios-9 MA flat-top, $15 \mathrm{MA}$ L-mode and $15 \mathrm{MA} \mathrm{H}$-mode flat-top) the outer plasma edge in the $Z=0$ plane is displaced by several centimetres, shown in figure 28. The toroidal dependence of the displacement is not a pure $n=3$ or $n=4$ due to the rather impure spectrum applied from the IVCCs together with the TF ripple. For the $n=4$ RMPs with $I_{\mathrm{IVCC}}=90 \mathrm{kAt}$ in the $9 \mathrm{MA}$ plasma, the plasma edge is displaced by up to $\approx 5 \mathrm{~cm}$ from its axisymmetric position. The smallest edge displacement is observed in the 15 MA L-mode scenario, for both $n=3$ and $n=4$ RMPs, where the corrugation is roughly $1 \mathrm{~cm}$ if $I_{\mathrm{IVCC}}=90 \mathrm{kAt}$. Pertinently, the $15 \mathrm{MA}$ cases with $I_{\mathrm{IVCC}}=90 \mathrm{kAt}$ in $n=4$ configuration, as envisaged for baseline $Q=10$ operation, has a midplane displacement of $\pm 2 \mathrm{~cm}$ (or $\pm 1 \%$ of the minor radius).

\subsection{Resistive MHD modelling}

Finally, nonlinear resistive MHD simulations have also been performed for the 15 MA ITER baseline scenario with two different codes: M3D-C ${ }^{1}$ and JOREK. In principle, these models represent the most accurate representation of the empirical situation and should therefore give greatest fidelity to the experiment and have greatest weight in the prediction for ITER, although the uncertainties in various parameters and 

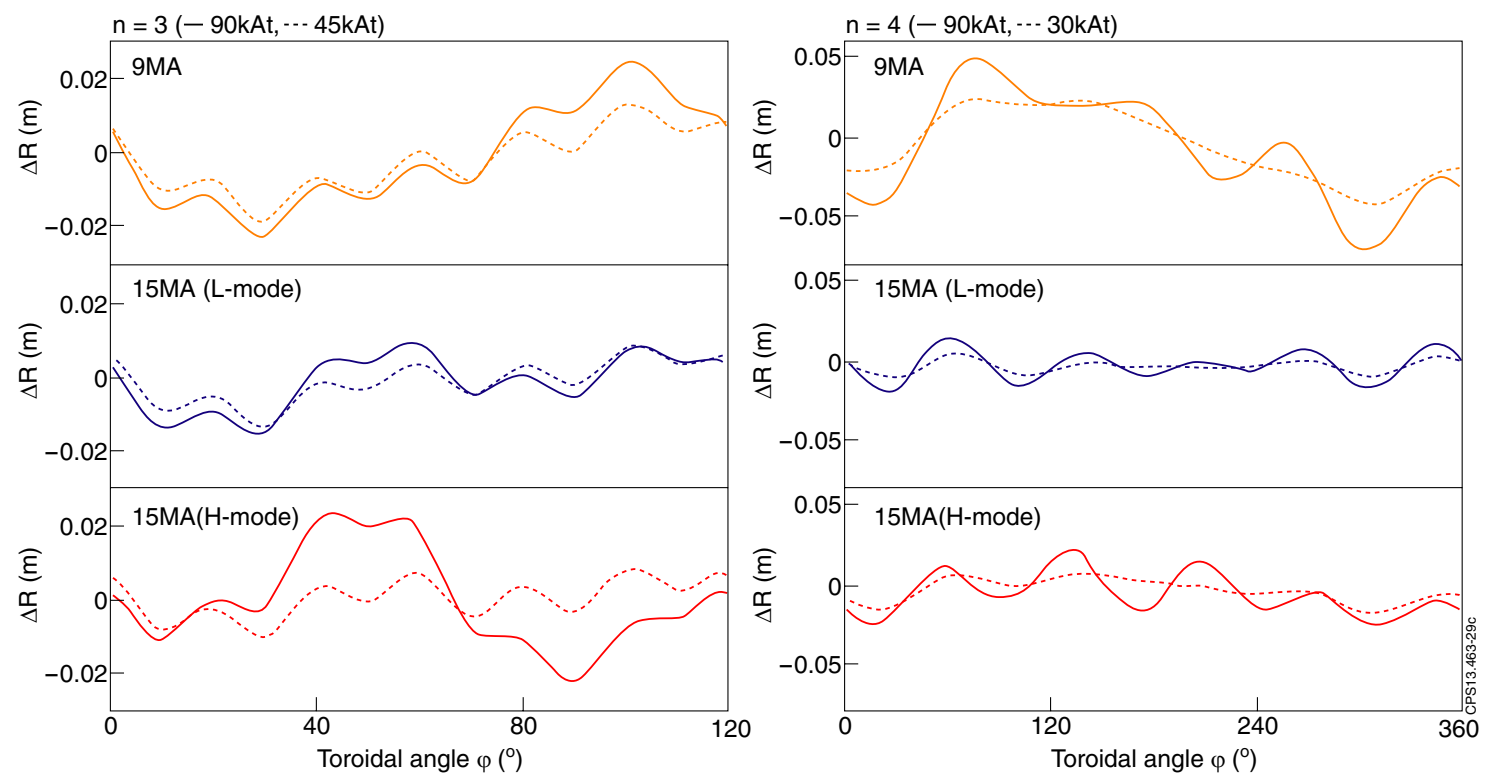

Figure 28. ITER. The midplane plasma boundary displacement as predicted by NEMEC for the (top pane) ITER 9 MA scenario, (centre pane) 15 MA L-mode phase and (bottom pane) $15 \mathrm{MA} \mathrm{H}$-mode phase as modelled with CORSICA. All 12 calculations are shown. The $90 \mathrm{kAt}$ calculations are indicated by solid lines, the calculations with $45 \mathrm{kAt}$ in the $n=3$ cases (left column) and the results with $30 \mathrm{kAt}$ in the $n=4$ cases (right column) by dashed lines.
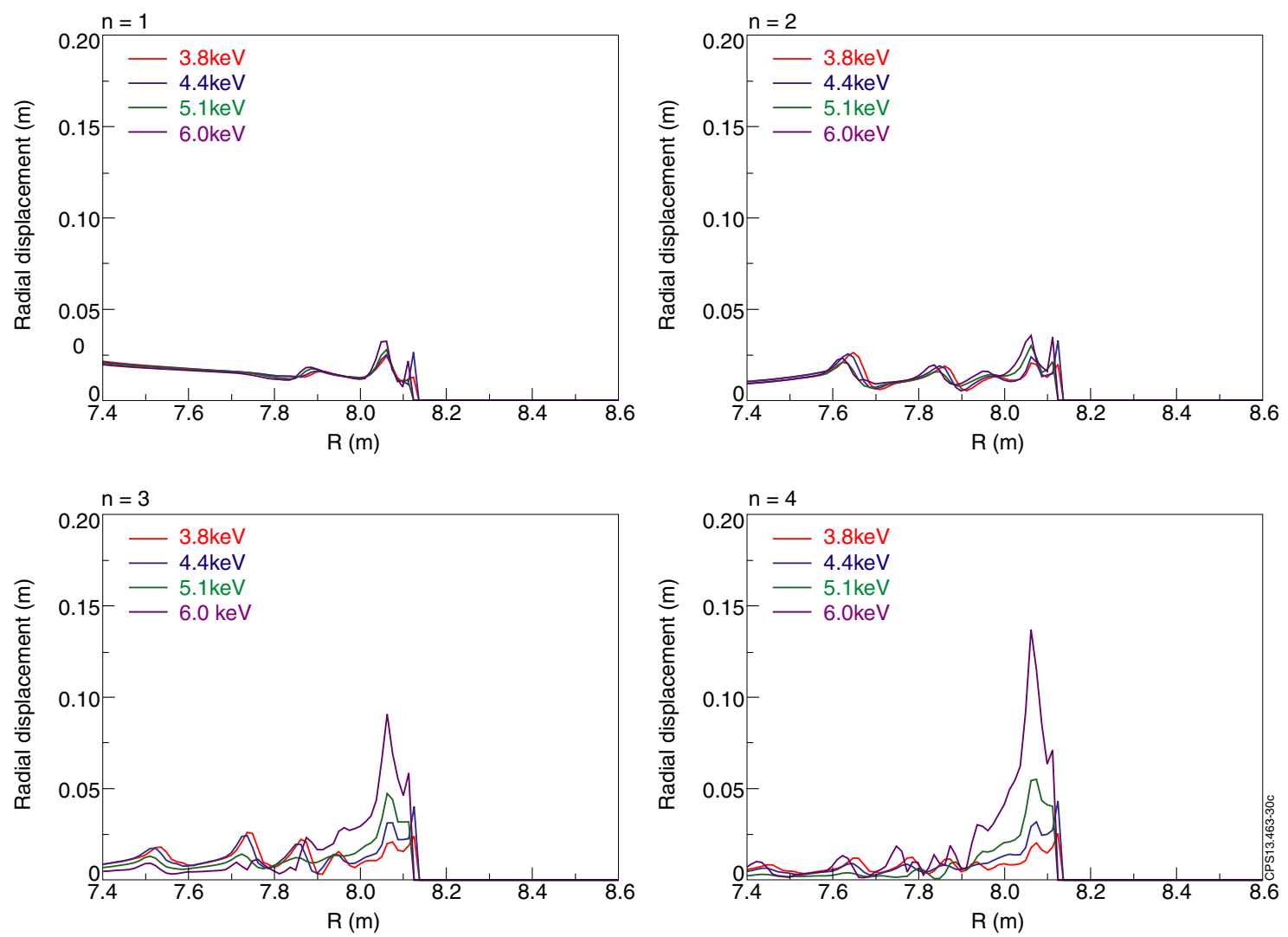

Figure 29. ITER. The radial displacement as a function of the major radius in ITER baseline H-mode scenario as predicted by linear simulations by the M3D-C code when $90 \mathrm{kAt}$ is applied from the in-vessel coils in $n=1,2,3,4$ configurations, showing increasing displacement with pedestal temperature.

profiles which influence the results strongly means that the prediction for ITER still comes with significant uncertainty.

Figure 29 shows the radial displacement across the pedestal region when $n=1,2,3,4$ RMPs are applied with maximum amplitude (i.e. $I_{\mathrm{IVCC}}=90 \mathrm{kAt}$ ) as predicted by linear M3D-C ${ }^{1}$ simulations assuming various values for the pedestal-top temperature [42]. In all cases the boundary displacement increases with the pedestal temperature as the 

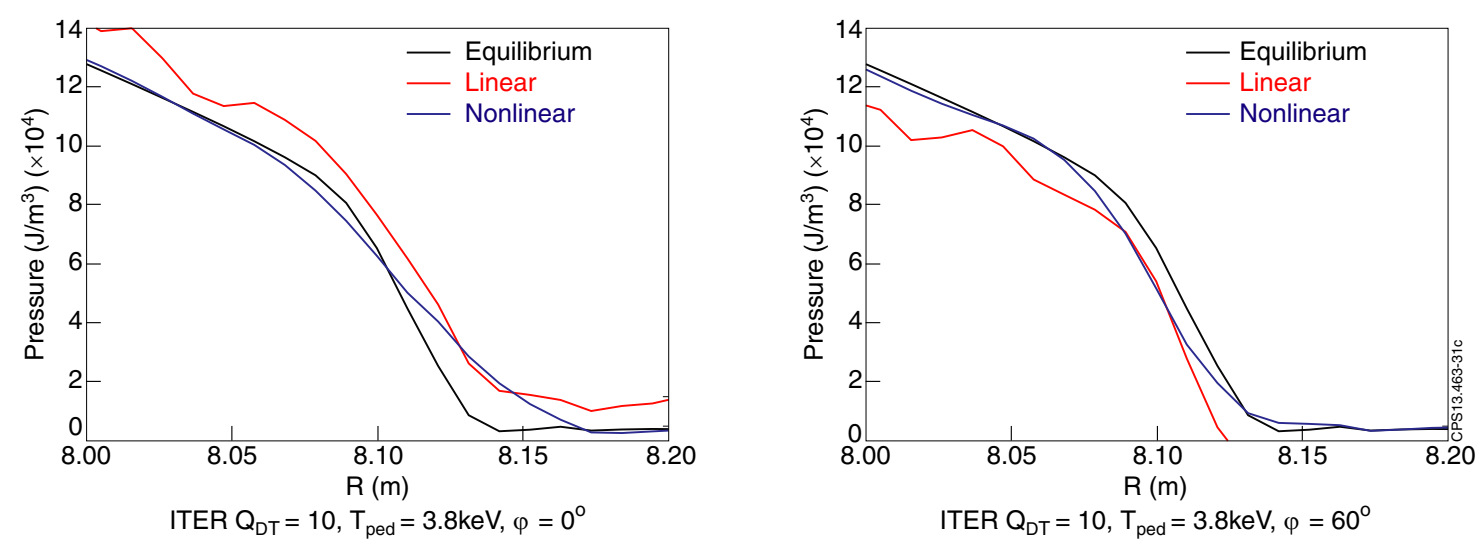

Figure 30. ITER. The pressure predicted by the linear and nonlinear response calculations with M3D-C ${ }^{1}$ for ITER baseline scenario with a $3.8 \mathrm{keV}$ pedestal and an $n=3$ field applied at full coil current. The two figures show the pressure profile in two toroidal positions $60^{\circ}$ apart.

applied field is amplified by increasingly unstable low- $n$ peeling modes at the plasma edge. For the $n=3$ and $n=4$ configurations (as intended for ELM control) the boundary displacement can exceed $5 \mathrm{~cm}$ for high pedestal pressures. However, it is important to note that the overlap criterion for the validity of linear simulations, $\left|\mathrm{d} \xi_{r} / \mathrm{d} r\right|<1$ (more details of which can be found in [42]) is violated for $T_{\text {e,ped }}=5.1,6.0 \mathrm{keV}$ [42]. Nonetheless, even at $T_{\text {e,ped }}=4.4 \mathrm{keV}$, which is assumed as the operating point for $Q=10$ baseline scenario [68], both the $n=3$ and $n=4$ RMPs lead to a boundary displacement of $\pm \xi_{a}=3.5 \mathrm{~cm}$, or $\pm 1.75 \%$ of the minor radius.

Figure 30 shows a comparison of the pressure predicted by the linear and nonlinear response calculations with M3D-C ${ }^{1}$ at $Z=0$ at two different toroidal positions in ITER baseline scenario. In this case a $3.8 \mathrm{keV}$ pedestal is assumed with an $n=3$ RMP applied at full coil current. Both calculations used the same transport coefficients and mesh and both are single-fluid. The linear and nonlinear simulations give similar pressure profiles, and importantly both show the same direction of the boundary displacement in each toroidal position, though the amplitude of the displacement varies. The difference seems to be that the nonlinear calculation develops significant $n<3$ response (the applied field is dominantly $n=3$ with some $n=6$ sideband). If the response were purely $n=3$, one would expect that the boundary displacement should be equal and opposite in the two figures; this is (exactly) true for the linear calculation, but it is clearly not the case for the nonlinear calculation. Nonetheless, this comparison suggests that the linear calculations give a good approximation for the boundary displacement (at least for the $3.8 \mathrm{keV}$ pedestal which is well below marginal stability), albeit neglecting small corrections from $n \neq 3$ components. In this case, the boundary displacement at the midplane when an $n=3$ field is applied is $\pm 2 \mathrm{~cm}$, equating to $\pm 1 \%$ of the minor radius.

The JOREK non-linear MHD code has also been used to simulate the effects of RMPs in ITER [43]. Figure 31 shows a Poincare plot of the magnetic field lines in ITER baseline scenario when diamagnetic flows and realistic toroidal rotation (left) are not included and (right) are included. The displacements near the X-point are significantly radially extended, but the boundary displacements away from the Xpoint are negligible. Figure 32 shows the electron density and temperature profiles across the pedestal as predicted by

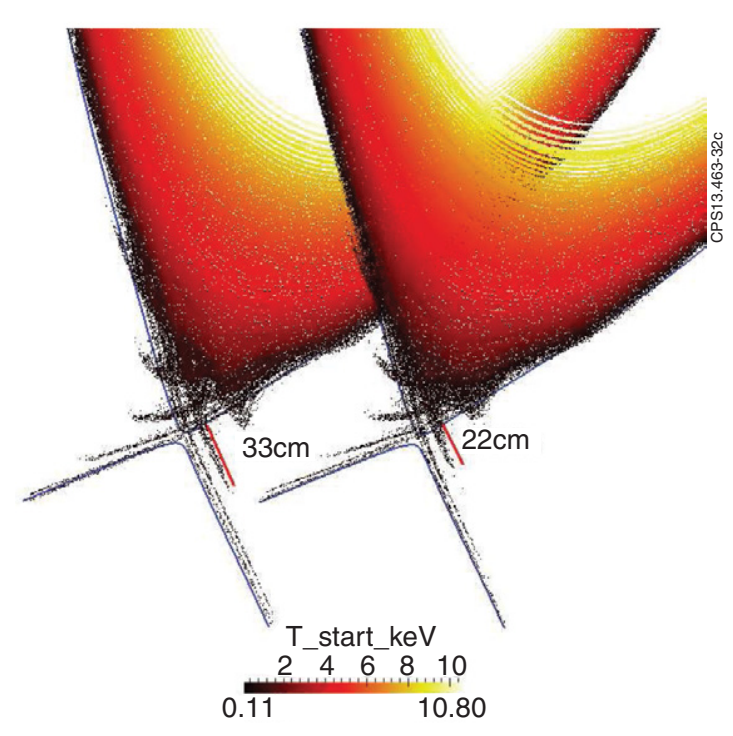

Figure 31. ITER. A Poincare plot of the magnetic field lines in ITER baseline scenario when diamagnetic flows and realistic toroidal rotation (left) are not included and (right) are included in JOREK, showing large displacements near the X-points. Reproduced with permission from Orain F. et al 2013 Phys. Plasmas 20 102510. Copyright 2013 by AIP Publishing LLC.

JOREK when an $n=3 \mathrm{RMP}$ is applied with $I_{\mathrm{IVCC}}=55 \mathrm{kAt}$, illustrating a boundary corrugation of $\pm 1 \mathrm{~cm}$, i.e. only $\pm 0.5 \%$ of the minor radius [43]. Of course this would be expected to approximately double at full applied field, in excellent agreement with the M3D-C ${ }^{1}$ results for the same ITER case shown in figure 30 , adding credence to this prediction.

\subsection{Overview of ITER modelling}

A range of numerical models have been used to predict the displacements likely in ITER due to the application of RMPs. Vacuum field line modelling suggests the separatrix deformation will be $\pm 3 \mathrm{~cm}$, equating to $\pm 1.5 \%$ of the minor radius. Of course, this assumes no plasma screening nor 


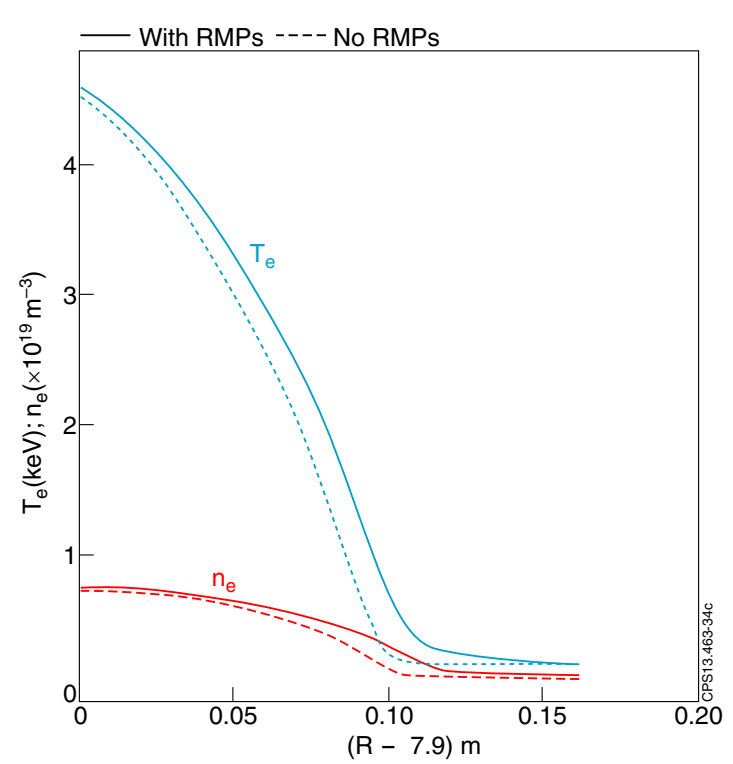

Figure 32. ITER. The electron density and temperature as a function of the minor radius as predicted by a non-linear JOREK simulation when $55 \mathrm{kAt}$ is applied in $n=3$ configuration. Reproduced with permission from Orain F. et al 2013 Phys. Plasmas 20 102510. Copyright 2013 by AIP Publishing LLC.

amplification. More sophisticated ideal 3D equilibrium modelling predicts that, for the $15 \mathrm{MA}$ baseline scenario, both $n=3$ and $n=4$ RMPs at full applied field will give rise to $\pm 2 \mathrm{~cm}$ midplane displacements. This modelling still assumes no screening and does not allow for formation of magnetic islands. Resistive nonlinear MHD modelling using two independent codes, which provide the most complete physical model of the plasma, predicts that for $T_{\text {e,ped }}=$ $3.8 \mathrm{keV}$, one could expect a midplane boundary displacement of $\pm 2 \mathrm{~cm}$. The amplitude of this corrugation increases as the temperature pedestal increases, or as the plasma flows decrease. For $T_{\mathrm{e} \text {,ped }}=4.4 \mathrm{keV}$, as assumed for $Q=10$ operation $[66,68]$, the displacement amplitude is $\pm 3.5 \mathrm{~cm}$, comparable to the prediction assuming vacuum field only.

\section{Discussion and conclusions}

It is evident that applied non-axisymmetric fields can give rise to significant displacements of the plasma boundary. These displacements have been measured in various tokamaks and successfully compared with numerical simulation. A multi-machine database exhibits a linear correlation between the measured corrugation and the resonant applied field as predicted by vacuum modelling, suggesting that vacuum field line tracing can at least qualitatively replicate the empirical phenomenology. Indeed, in the case of sub-cm displacements in ASDEX Upgrade, NSTX and DIII-D vacuum models agree quantitatively. However, there are cases in JET, MAST and DIII-D where vacuum modelling significantly under-predicts the corrugation. In these cases, ideal threedimensional equilibrium modelling provides greater fidelity with experimental measurements. Even so, there are some empricial measurements at very large displacement-for example the displacements of up to $5 \mathrm{~cm}$ observed in JETwhere only resistive nonlinear MHD models can accurately replicate measurements. Such models including plasma screening, amplification, flows and allowing islands to form are the most representative tools and can reproduce measured corrugations over parameter scans in various machines. The RMP-induced displacements foreseen in ITER are expected to lie within the range of those predicted by the different models, meaning less than $\pm 1.75 \%( \pm 3.5 \mathrm{~cm})$ of the minor radius in the $\mathrm{H}$-mode baseline and less than $\pm 2.5 \%( \pm 5 \mathrm{~cm})$ in a $9 \mathrm{MA}$ plasma.

Such toroidal corrugation of the plasma boundary affects many things, notably the coupling of ICRH, the minimum values of wall gaps assumed for safe operation, the plasma position control, and (de facto) the control of ELMs. Indeed, such a boundary corrugation may be necessary to affect the ELMs [21] and using plasma control feedback to provide an $n>0$ correction may compromise the efficacy of the RMPs as an ELM control scheme. Whilst a displacement of $\pm 3 \mathrm{~cm}$ in the baseline scenario is allowable from both a plasma control and heat loading perspective, it is important that ITER adopts a plasma control system which can account for a threedimensional boundary corrugation to avoid an $n=0$ correction which would otherwise exacerbate the displacement caused by the applied fields.

\section{Acknowledgments}

This work was conducted in part under the auspices of the ITPA MHD Stability Topical Group. This work was partfunded by the RCUK Energy Programme [grant number EP/I501045]; the European Communities under the contract of Association between EURATOM and CCFE, CEA, IPP, CRPP, FZJ and VR; and US Department of Energy under DE-AC02-09CH11466, DE-FG02-07ER54917, DE-AC0500OR22725，DE-FC03-04ER54698，DE-FG02-03ER54692 and DE-FG02-95ER54309. To obtain further information on the data and models underlying this paper please contact PublicationsManager@ccfe.ac.uk. The views and opinions expressed herein do not necessarily reflect those of the European Commission or the ITER Organization. This work was carried out within the framework of the European Fusion Development Agreement.

\section{References}

[1] Connor J.W. 1998 Plasma Phys. Control. Fusion 40531

[2] Snyder P.B. et al 2002 Phys. Plasmas 92037

[3] Wilson H.R. et al 2006 Plasma Phys. Control. Fusion 48 A71

[4] Suttrop W. 2000 Plasma Phys. Control. Fusion 42 A1

[5] Loarte A. et al 2003 Plasma Phys. Control. Fusion 451594

[6] Evans T. et al 2004 Phys. Rev. Lett. 92235003

[7] Evans T. et al 2008 Nucl. Fusion 48024002

[8] Jeon Y.M. et al 2011 Proc. 53rd Annual Meeting of Division of Plasma Physics (Salt Lake City, USA) (New York: AIP) T04.00005 www.aps.org/units/dpp/meetings/ meeting.cfm?name $=$ DPP11

[9] Suttrop W. et al 2011 Phys. Rev. Lett. 106225004

[10] Suttrop W. et al 2011 Plasma Phys. Control. Fusion 53124014

[11] Moyer R.A. et al 2005 Phys. Plasmas 12056119

[12] Kirk A. et al 2012 Phys. Rev. Lett. 108255003

[13] Kirk A. et al 2013 Nucl. Fusion 53043007

[14] Kirk A. et al 2013 Plasma Phys. Control. Fusion 55015006

[15] Liang Y. et al 2010 Nucl. Fusion 50025013

[16] Liang Y. et al 2013 Nucl. Fusion 53073036 
[17] Saarelma S. et al 2011 Plasma Phys. Control. Fusion $\mathbf{5 3} 085009$

[18] Snyder P.B. et al 2007 Nucl. Fusion 47961

[19] Osborne T.H. et al 2008 J. Phys.: Conf. Ser. 123012014

[20] Chapman I.T. et al 2012 Nucl. Fusion 52123006

[21] Chapman I.T. et al 2013 Phys. Plasmas 20056101

[22] Lao L. et al 2005 Proc. 47th Annual Meeting of Division of Plasma Physics (Denver, USA) (New York: AIP) CP1.00034 http://meetings.aps.org/meeting/DPP05/Content/251

[23] Fischer R. et al 2011 'Effect of non-axisymmetric magnetic perturbations on profiles at ASDEX Upgrade' 38th EPS Conf. on Plasma Physics (Strasbourg, France) P1.072 and http://ocs.ciemat.es/EPS2011PAP/pdf/P1.072.pdf

[24] Fischer R et al 2012 Plasma Phys. Control. Fusion 54115008

[25] Fuchs J.C. et al 2011 Influence of non-axisymmetric magnetic perturbations on the equilibrium reconstruction at ASDEX Upgrade 38th EPS Conf. on Plasma Physics (Strasbourg, France) P1.090 and http://ocs.ciemat.es/EPS2011PAP/ pdf/P1.090.pdf

[26] Chapman I.T. et al 2007 Nucl. Fusion 47 L36

[27] Chapman I.T. et al 2012 Plasma Phys. Control. Fusion 54105013

[28] Lanctot M.J. et al 2011 Phys. Plasmas 18056121

[29] Moyer R.A. et al 2012 Nucl. Fusion 52123019

[30] Lanctot M.J. et al 2010 Phys. Plasmas 17030701

[31] Fundamenski W. and Pitts R. 2007 J. Nucl. Mater. 319363

[32] Kocan M. 2013 private communication

[33] Lister J.B., Portone A. and Gribov Y. 2006 Control Syst. 2679

[34] Yadykin D. et al 2014 Nucl. Fusion 54013016

[35] Urano H. et al 2011 Nucl. Fusion 51113004

[36] Saibene G. et al 2007 Nucl. Fusion 47969

[37] Frassinetti L. et al 2012 Rev. Sci. Instrum. 83013506

[38] Nardon E. 2007 Edge localized modes control by resonant magnetic perturbations PhD Thesis Ecole Polytechnique and www.imprimerie.polytechnique.fr/Theses/Files/ Nardon.pdf

[39] Hirshman S.P., van Rij W.I. and Merkel P. 1986 Comput. Phys. Commun. 43143

[40] Canik J.M. et al 2012 Nucl. Fusion 52054004

[41] Bird T.M. and Hegna C.H. 2013 Nucl. Fusion 53013004

[42] Ferraro N.M. et al 2013 Nucl. Fusion 53073042

[43] Becoulet M. et al 24th IAEA Fusion Energy Conf. (San Diego, CA) TH/2-1 www-pub.iaea.org/iaeameetings/41985/ 24th-Fusion-Energy-Conference

[44] Ferraro N.M. 2012 Phys. Plasmas 19056105

[45] Lazerson S.A. et al 2013 Nucl. Fusion submitted
[46] Spong D.A. et al 2011 Nucl. Fusion 41711

[47] Liu Y.Q. et al 2010 Phys. Plasmas 17122502

[48] Lazerson S.A. et al 2012 39th EPS Conf. on Plasma Physics (Stockholm, Sweden) http://epsicpp2012.spp.ee.kth.se/

[49] Orlov D. et al 2014 Proc. 53rd Annual Meeting of Division of Plasma Physics (Providence, USA) (New York: AIP) Poster GP8.77 Nucl. Fusion (submitted) http://meeting.aps.org/Meeting/DPP12/Content/2392

[50] Huysmans G.T.A. et al 2009 Plasma Phys. Control. Fusion 51124012

[51] Daly E. et al 2013 Fusion Sci. Technol. 64168

[52] Schaffer M.J. et al 2008 Nucl. Fusion 48024004

[53] Becoulet M. et al 2008 Nucl. Fusion 48024003

[54] Lazerson S.A. et al 2014 Three dimentional equilibrium reconstruction on the DIII-D device Nucl. Fusion submitted

[55] Fenstermacher M.E. et al 2008 Phys. Plasmas 15056122

[56] Evans T.E. et al 2013 Nucl. Fusion 53093029

[57] Evans T.E., Moyer R.A. and Monat P. 2002 Phys. Plasmas 94957

[58] Evans T. et al 2005 J. Phys.: Conf. Ser. 7174

[59] Wingen A. et al 2009 Nucl. Fusion 49055027

[60] Evans T.E. 2008 Implications of topological complexity and Hamiltonian chaos in the edge magnetic field of toroidal fusion plasmas Chaos, Complexity and Transport: Theory and Applications ed C Chandre et al (Singapore: World Scientific) pp 147-76, ISBN-13 978-981-281-897-9

[61] Jakubowski M.W. et al 2009 Nucl. Fusion 49095013

[62] Nardon E. et al 2011 J. Nucl. Mater. 415 S914

[63] Cahyna P. et al 2011 J. Nucl. Mater. 415 S927

[64] Schmitz O. et al 2008 Plasma Phys. Control. Fusion $\mathbf{5 0} 124029$

[65] Liu Y.Q. et al 2011 Nucl. Fusion 51083002

[66] Casper T. et al 2012 24th IAEA Fusion Energy Conf. (San Diego, CA) ITR/P1-15 www-pub.iaea.org/ iaeameetings/41985/24th-Fusion-Energy-Conference

[67] Gribov Y. and Loarte A. 2012 Curents in ELM coils for edge plasma shape perturbation studies Technical Report ITER 98BA5X

[68] Saarelma S. et al 2012 Nucl. Fusion 52103020

[69] Lazerson S.A. and Chapman I.T. 2013 Plasma Phys. Control. Fusion 55084004

[70] Orain F. et al 2013 Phys. Plasmas 20102510

[71] Harrison J.R. et al 2013 Characteristics of X-point Lobe structures in single-null discharges on MAST Nucl. Fusion at press 